SAND75-0248A

Unlimited Release

UC-71

\title{
Transportation Environment Data Bank Index
}

Clarence A. Davidson, Jerry T. Foley

Prepared by Sandia Laboratories, A lbuquerque, New Mexico 87115

and Livermore, California 94550 for the United States Energy Research

and Development Administration under Contract AT (29-1)-789

Printed February 1976

Supersedes SAND75-0248

\section{Sandia Laboratories


Issued by Sandia Laboratories, operated for the United States Energy Research and Development Administration by Sandia Corporation.

\section{NOTICE}

This report was prepared as an account of work sponsored by the United States Government. Neither the United States nor the United States Energy Research and Development Administration, nor any of their employees, nor any of their contractors, subcontractors, or their employees, makes any warranty, express or implied, or assumes any legal liability or responsibility for the accuracy, completeness or usefulness of any information, apparatus, product or process disclosed, or represents that its use would not infringe privately owned rights. 


\section{DISCLAIMER}

This report was prepared as an account of work sponsored by an agency of the United States Government. Neither the United States Government nor any agency Thereof, nor any of their employees, makes any warranty, express or implied, or assumes any legal liability or responsibility for the accuracy, completeness, or usefulness of any information, apparatus, product, or process disclosed, or represents that its use would not infringe privately owned rights. Reference herein to any specific commercial product, process, or service by trade name, trademark, manufacturer, or otherwise does not necessarily constitute or imply its endorsement, recommendation, or favoring by the United States Government or any agency thereof. The views and opinions of authors expressed herein do not necessarily state or reflect those of the United States Government or any agency thereof. 


\section{DISCLAIMER}

Portions of this document may be illegible in electronic image products. Images are produced from the best available original document. 
SAND75-0248A

Unlimited Release

Printed February 1976

Supersedes SAND75-0248
Distribution

Category UC-71

TRANSPORTATION ENVIRONMENT DATA BANK INDEX

\author{
Clarence A. Davidson \\ Jerry T. Foley \\ Environmental Analysis Division 1285 \\ Sandia Laboratories \\ Albuquerque, NM 87115
}

\begin{abstract}
In an effort to determine the environment intensities to which shipping containers will be exposed, a "Data Bank" of environmental information has been established by Sandia Laborator ies, Division 1285 for the ERDA Division of Environmental Control Technology. This document is an index which can be used to request data of interest.
\end{abstract}

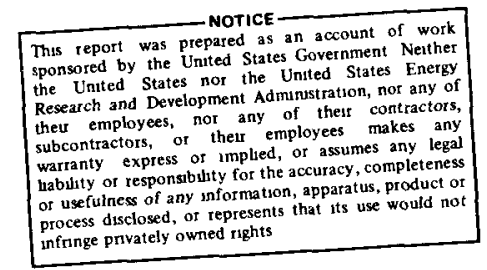

Printed in the United States of America

Available from

National Technical Information Service

U S Department of Commerce

5285 Port Royal Road

Springfield, Virginis 22161
Price Printed Copy $\$ 500$, Microfiche $\$ 2,25$ 
0

• 
Int roduction

Page

The Environments

The Data

7

Mechanics of the Data Bank

10

l)ata Sources

10

Avallabsluty of the Data

11

'H Io' Indos

11

12

Normal Environments

13

Abnormal Environments

25 
-

• 
The purpose of the Transportation Environment Data Bank is to store and make available descriptions of the environment of transportation expressed in engineering terms. The data are intended to provide environmental criterıa to shipping container design and test engineers as a basis for their functions. The Transportation Environment Data Bank is a segment of a larger ERDA/DOD environmental Data Bank for weapons application.

For purposes of indexing and data retrieval, the data are catalogued under two major headıngs: Normal and Abnormal Environments. The diagram below lllustrates the relationship between these environmental designations.

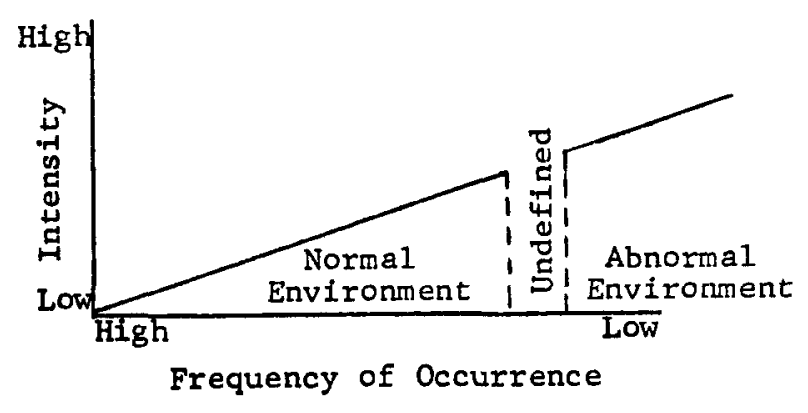

Normal environments are those which will be encountered at some level during every shipment. They have a high frequency of occurrence, but a relatively low intensity. The abnormal or accident environments, on the other hand, are characterized by higher intensities but lower frequency of occurrence. The very high intensities of environment occur very infrequently. The abnormal environments, whlle often called by different names to differentiate them, have actually the same engineering parameters as the normal. Thus, an acceleration is experienced by cargo as a truck crosses rallroad tracks at $50 \mathrm{mph}$. A greater acceleration may result if it strikes a bridge abutment at the same speed. To make the difference clear in a succinct manner, the latter is termed, "impact." These short descriptions are shown in the index in parentheses where they apply.

\section{The Environments}

Twelve categories of environment have been identified as relevant to transportation. These include: 
1. Acceleration/Time

2. Acoustic Nolse

3. Atmospheric Contents

4. Fragmentation ${ }^{*}$

5. Humidity

6. Precipitation
7. Pressure *

8. Radiation ${ }^{*}$

9. Shock*

10. Temperature ${ }^{*}$

11. Vibration

12. Wind ${ }^{*}$

Three factors operate to limit the number of these environments for which abnormal levels require consideration:

1. Some environments reach an absolute limit regularly, and the limit thus is within the normal range;

2. The abnormal aspects of some of them are encountered so infrequently as to be of little interest; and

3. Protection against the effects of some environments include protection against the abnormal.

After eliminating from consideration those listed environments falling within the above limitations, only the environments indicated by an asterisk in the above list are of interest in the abnormal considerations. Figure 1 presents the environments which relate to transportation and the detalls of the abnormal environments as to input and response.

Input may be defined as the environmental level which exists at the cargo interface, while response is considered as the way in which the cargo reacts to the environments. As an example, consider the pressure environment. Pressure has both normal and abnormal levels. The normal, which all cargo will experience, includes the varıatıons in atmospheric pressure at a specific location, as well as the variations produced by changes in elevation, both on the earth's surface and while flying. The abnormal aspects of the pressure environment are the result of exposure to an explosion or to immersion (inputs); if the intensity level of input is sufficiently high, a crush response of the cargo will result.

Four of the environments are considered only in the normal aspects for reasons mentıoned above. One environment, fragmentation, is considered only in the abnormal aspect. This is because no elements which will cause puncture to the cargo are present under normal conditions, $1 . e$. , will be encountered frequently during transportation.

\footnotetext{
"Environments with both normal and abnormal aspects.
} 


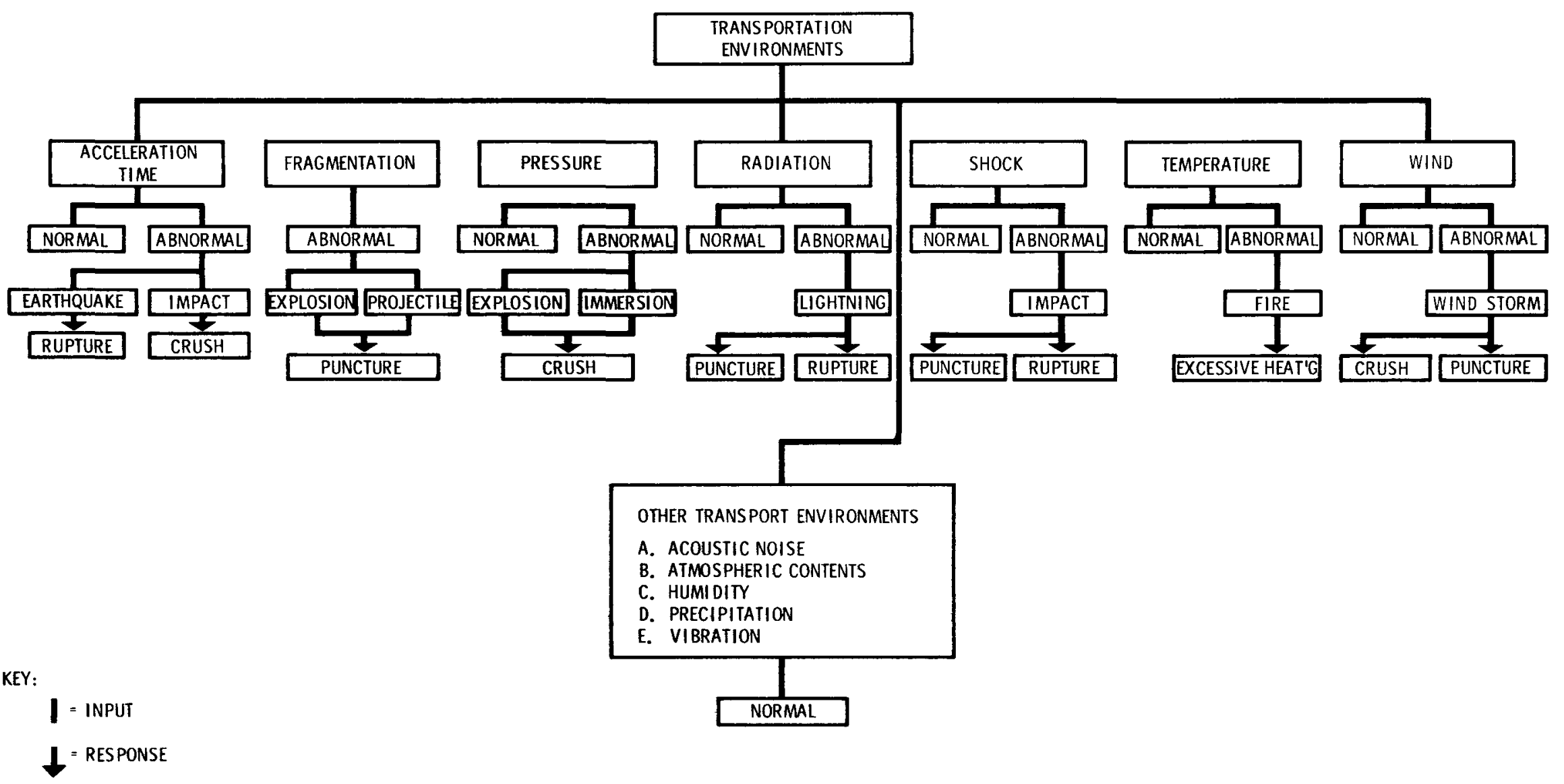

Figure 1. Structure of the Transportation Environment Data Bank 
The Data

Both input and response data contaıned in the Transportation Environment Data Bank relatıng to the normal environments are measured data. They were obtained from reliable sources, both withın Sandia Laboratories and from other reputable agencies. Wnen data were required, but not avallable, the Data Bank operators arranged for measurements to provide the information.

Most input data in the Data Bank under the heading of Abnormal Environments, however, were not measured directly. Because of the number and randomness of occurrence and type, it $151 \mathrm{mpos}-$ sible to provide engineering instrumentation for measurement. If an instrumented vehicle is subjected to a staged accident, it represents only the one type of event. Therefore, it is necessary to use statistical techniques to characterize the range of environmental intensities which may be experienced by cargo in accidents. The data to which these techniques are applied are often estimated. The basic data which are most useful to estimate abnormal inputs include the tollowing.

Abnormal Input
1. Earthquake
2. Impact
3. Explosion
4. Projectile/Impalement
5. Immersion
6. Lightning
7. Fire
8. Windstorm

Useful Data Parameters
Time History
Vehicle Velocity
Pressure or Missile Velocity, Radius of Missile Travel
Package Velocily, Radius of Probe
Depth
Voltage, Amperage, Rise T me, etc.
Ternperature, Fle.t Flux an 1 Duration
Velocity, Pressure Differential

It is these types of data, or information which permit the derivatic $n$ of the se values, which are stored in the input section of the Abnormal Environment part of he Transfortation Environment Data Bank. The response section of the Bank is expressed in terms of damage to cargo, Since the damage can be described and measured, measurements and descriptions are included wherever possible.

Mechanıcs of the Data Bank

The data which have been retrieved and entered in the Data Bank are on microfulm. Two forms of microfllm are used: aperture cards and microfiche. Facllities for viewing the data and obtaining hard copy are located at the bank site. When processing large requests for information, the Bank's operators have access to automated means for obtalnung hard copy.

When either raw data or published information is acquired, is is reviewe 1 for engineering data content. Pertinent information is then extracted, collated, and microfllmed. The data cards and/ or microfiche are filed in numerical order without regard to subject. This siriple technique is 
possible because of a computerized indexing and retrieval program which was developed for effic1ent use of the Data Bank.

The use of a computer-aided system makes it possible to obtain a more flexible file, permits more complete data retrieval, shortens the time necessary for search, and makes feasible more frequent publication of a current index.

Data Sources

Normal environmental information for inclusion in the Data Bank is acquired by the Data Bank's operators from many sources. Mllitary agencies, such as Aberdeen Proving Ground, Wright Air Development Center, Naval Ordnance Test Center, and Army QM R\&E Center, and industrial groups, such as rallroads, aircraft companies, and trucking concerns, have contributed information. A considerable portion of the information has been obtained from various project groups within Sandia Laboratories. The Data Bank's operators not only extract information from published sources, but engage in specific research activities to obtain data not otherwise avallable.

Abnormal environmental information is obtained primarily from U. S. Department of Transportation sources and reports produced by the National Transportation Safety Board. Contacts have been established with various individuals and agencies responsible for accident investigation in the various modes of transportation. An information sources file is maintained as an adjunct to the Bank which integrates thıs Data Bank's efforts with other Data Banks collectıng related accident information such as accident rates, fatalities, etc.

\section{Avallability of the Data}

The information in the Data Bank is avallable, within the limits of time, workload, and security regulations, to any requestor. Requests for data should be sent to Division 1285, Sandia Laboratories, Albuquerque, New Mexico, 87115. The request should either outline the specific area for which information is desired, or it should list specific entries, including serial numbers. Requests will be processed as time allows. Agencies outside of the ERDA complex wll be furnished information subject to the review and release procedures established by the ERDA. 
The Index

The index provided on the following pages is what the Bank's operators call a category index. Entry is made into this index by finding the environmental category of interest, such as shock, temperature, vibration, etc. The 12 categories are listed alphabetically.

The two-part number listed after the title of a particular Data Bank entry is the access number for the data on flle. The first number, e.g., 1614, is the file number. The number after the dash $(-)$ is the number of file cards avallable on the subject. Requests for hard copies of data should cite these two numbers. 
Normal Environments 
-...ACCELERATION/TIME.

INPUT.

AIRCRAFT . . . . .

NTSB 1974 REPORT TO CONGRESS, AIRCRAFT

UNC $\quad 01904-004+$

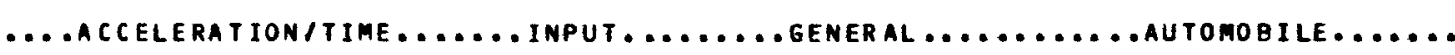

NTSB 1974 REPORT TO CONGRESS, AUTOMOBILE

UNC $\quad 01905-001+$

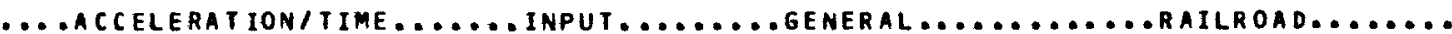

NTSB 1974 REPORT TO CONGRESS, RAIL

UNC 01906-001+

-...ACCELERAT TON/TIME.

- INPUT. . ... TRANSPORTATION.

IRCRAFT..........

LOADS IMPOSED ON CARGO BY FLIGHT AND LANDING UNC 00750-001*

MANEUVER LOAD DATA C-130 AIRCRAFT

FATIGUE DAMAGE PARAMETERS, TRANSPORT AIRCRAFT UNC $01085-001+$

GUST LOAD FREQUENCY, AIRCRAFT FLIGHTS,40-45KILOFEET, IN STORM UNC 01087-0024

AIRCRAFT RECORDINGS DURING TURBULENCE INCIDENT

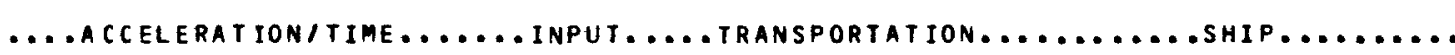

ACCELERATION,SHIP MOTIONS,MAXIMUM UNC 00739-001.

AC CELERATIONS ON PACKAGES STOREO IN SHIPS HOLOS UNC 00740-001+

ACCELERATION, EXPECTED PEAK VALUES, MARINE UNC 00741-001.

SHIP MOTIONS SS SILVER MARINER NEH YORK BREMERHAVEN, GERMAYY UY

SHIP MOTIONS T-2 TANKER,ESSO ASHVILLE, NORMAL OPERATIONS UNC $00746-001$.

U UNC 00747-001*

WAVE HEIGHT DISTRIBUTION - ATLANTIC COAST UNC 01573-004*

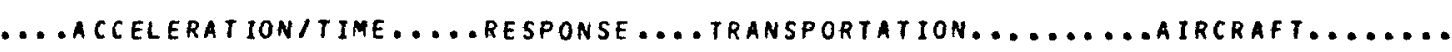

GUST LOAD FREQUENCY, AIRCRAFT FLIGHTS,40-45KILOFEET, IN STORM UNC 01087-002* AI RCRAFT RECORDINGS DURING A TURBULENCE INCIDENT UNC 01090-001+

(IMPACT)

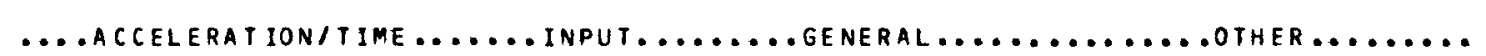

APPROXIMATE DURATION OF SOME SHORT ACCELERATION LOADS UNC 00734-001+

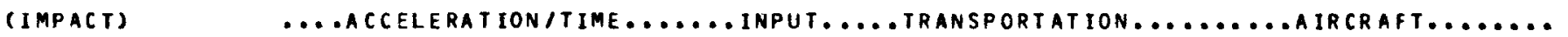

ARMY ROTARY WING AIRCRAFT ACCIDENTS-COST STUDY 1970-71 UNC 01895-016+ 


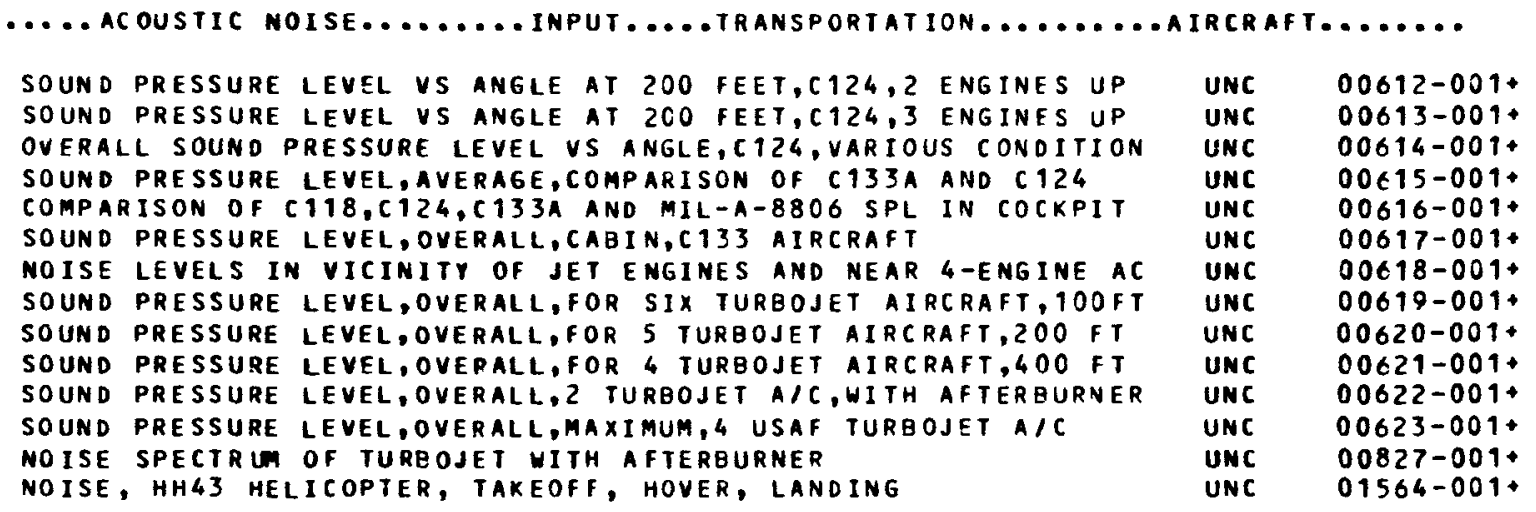

..... AC OUSTIC NOISE......... INPUT..... TRANSPORTATION........AUTOMOEILE......

$\begin{array}{lll}\text { CONTROL OF HIGHWAY NOISE } & \text { UNC } & 01134-003+ \\ \text { ACOUSTIC NOISE SOURCES, TRUCK } & \text { UNC } & 01606-001+\end{array}$

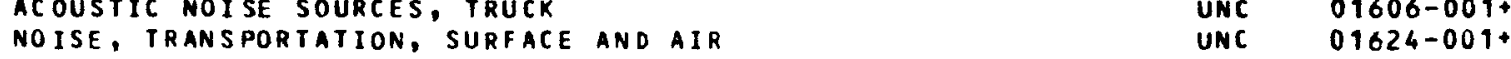

.. atmospheric contents...... input ........ general............ other........

ENVIRONMENT CONDITIONS PREVAILING DURING TRANSIT $\quad$ UNC 00078-001*

$\begin{array}{llll}\text { SALT SPRAY PARTICLES IN AIR.DISTRIEUTION, CONCENTRAIION,SIZE } & \text { UNC } & 00274-001+ \\ \text { SAND AND DUST-CHACTERISTICS } & \text { UNC } & 01274-002+\end{array}$

... Atmospheric contents...... input......... storage............ other........

AIRBORNE PARTICULATES

UNC $\quad 01568-001$

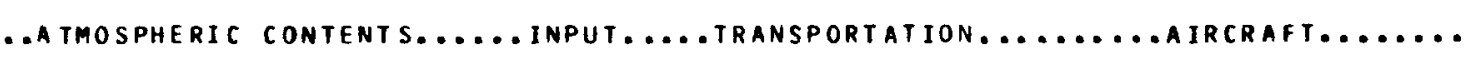

SAND/OUST IN HELICOPTERIVSTOL A/C FLIGHTS UNC 01084-005+

FREQUENCY OF OCCURENCE OF AIRCRAFT ICING PARAMETERS UNC 01089-002+

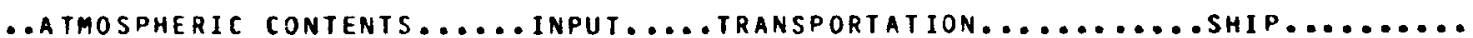

SEA SALT ENVIRONMENT ON DECK AT SEA

UNC $\quad 01179-002$ 


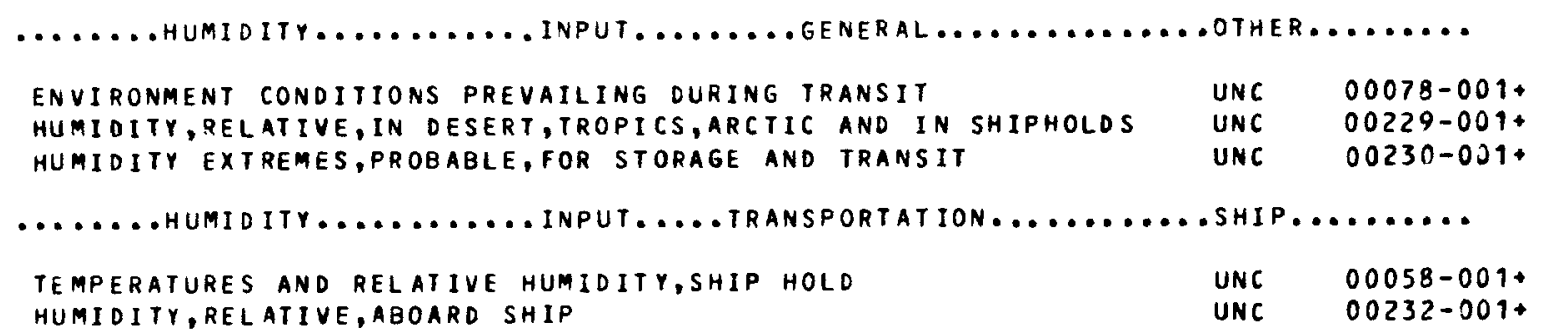

..... precipitation........ input ........

ENVIRONMENT CONDITIONS PREVAILING DURING TRANSIT P UNC 00078-001. RAINFALL, AVERAGE RATES OF $\quad$ UNC 00237-001+

HA ILSTONES,SIZE, TERMINAL VELOCITIES,DISTRIBUTION OF UNC 00250-001+ HAIL,FREQUENCY AND DISTRIBUTION OF,FLIGHT THRU THUNDERSTORM UNC O0251-001. ELECTROSTATIC FIELDS IN THE ATMOSPHERE UNC 00459-002+ RAINFALL INTENSITY,FREQUENCY,U.S.,SO STATES, PORTORICO UNC $01113-029+$ $\begin{array}{lll}\text { HAILSTONE EXTREMES } & \text { UNC } & 01513-001+ \\ \text { GLAIE ICE, WIND LOADS AT EARTHS SURFACE } & \text { UNC } & 01722-001+\end{array}$

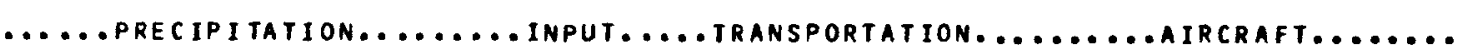

STATIC ELECTRICITY ACCUMULATION ON HELICOPTERS UNC 00463-001. FREQUENCY OF OCCURENCE OF AIRCRAFT ICING PARAMETERS UNC 01089-002+

...... Precipitation......... InPUt..... transportation..........

RAINFALL INTENSITY, DURATION, HAWAII

UNC $\quad 01761-001+$ 


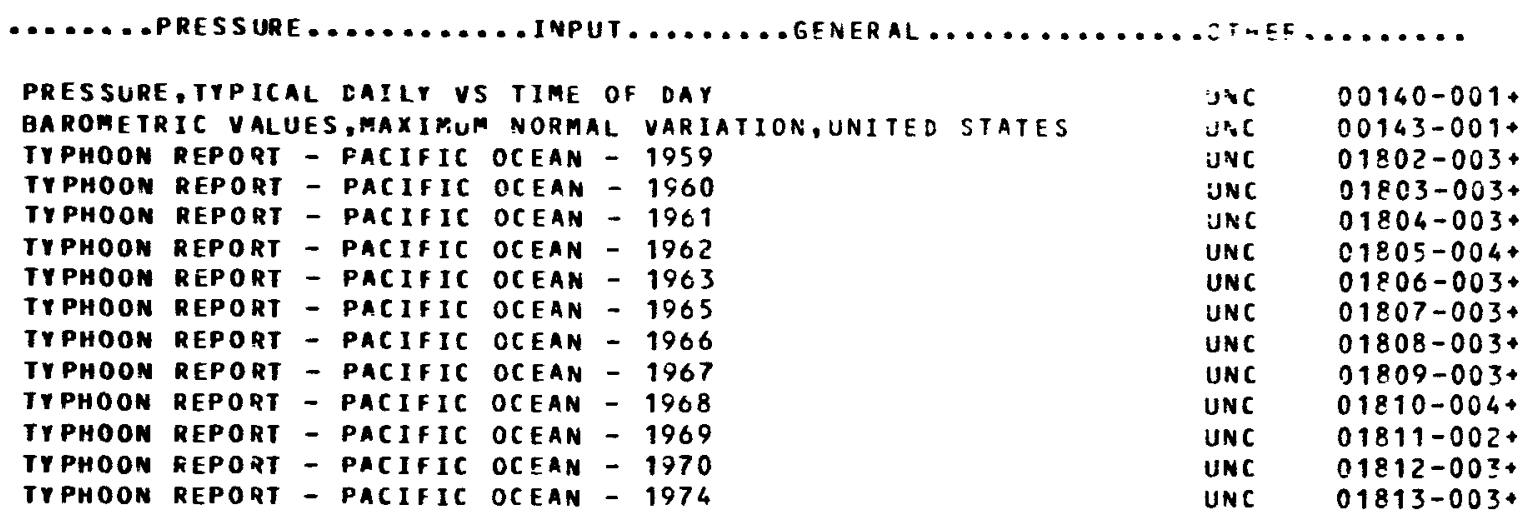

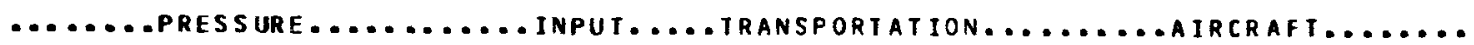

PRESSURE CHANGES,ATMOSPHERIC,AIRCRAFT CARGO HOLDS UNC 00147-001+ PERFORTANCE AND LOADING-C1308 UNC 00148-001+ PERFORMANCE AND LOADING-C133A

FLIGHT PROFILE, MORMAL, C133 CARGO AIRCRAFT UNC 00150-001+

FLIGHT PROFILE, NORMAL, C124 CARGO AIRCRAFT UNC 00151-001*

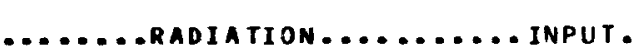

GENERAL

OTHER..........

ENVIRONMENT CONDITIONS PREVAILING DURING TRANSIT

UNC $\quad 00078-001+$ TE MP ERATURE, EXTREMESIDURATION, FOR STORAGE, HANDLING, TRANSPORT ELECTROSTATIC FIELDS IN THE ATMOSPHERE

STATIC ELECTRICITY-ELECTRICAL CHARACTERISTICS OF ATMOSPHERE RADIATION, SOLAR, AT EARTH S SURFACE AND ATMOSPHERE S EDGE GADIATION DATA, SOLAR

RADIATION, MEAN DAILY TOTAL, FLAT SURFACE, LOCATIONS, US /CANADA INSOLATION,MONTHLY MAPS OF MEAN DAILY, FOR THE UNITED STATES LIGHTNING CHARACTERISTICS

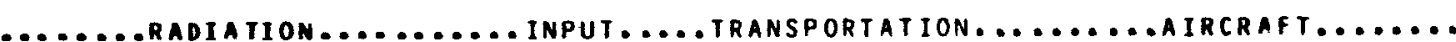

SIATIC ELECTRICITY ACCUMULATION ON HELICOPTERS UNC 00463-001+ HELICOPIER STATIC ELECTRICITY MEASUREMENTS UNC 00503-003*

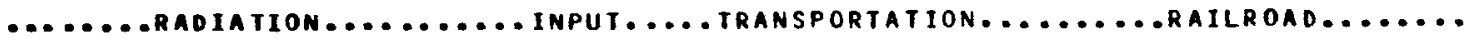

TEMPERATURE, INTERNAL AIR,RAILROAD EOXCAR IN DESERT

UNC $\quad 00056-001+$

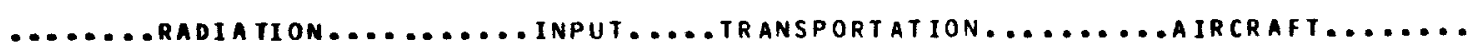




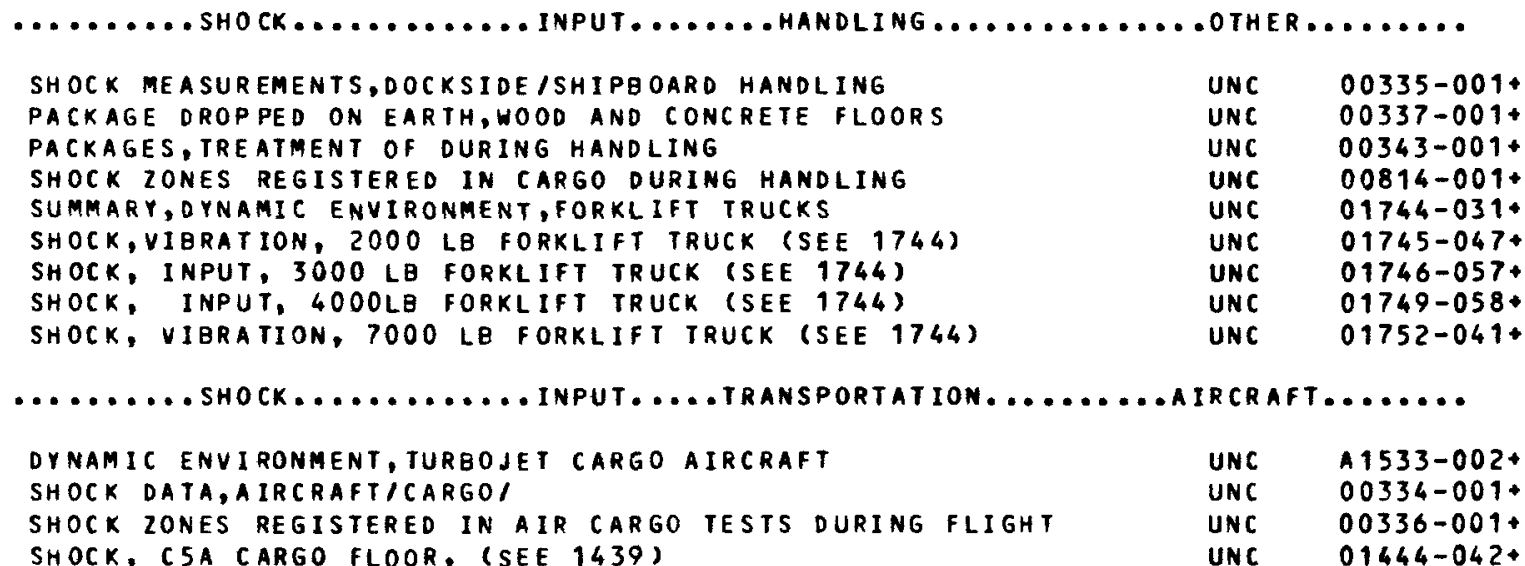

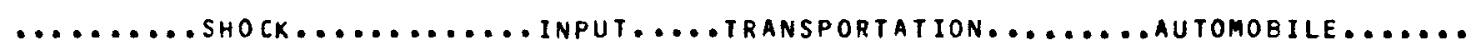

SHOCK DATA-TRUCKS

SHOCKS FROM ROADS $\quad$ UNC $00319-001+$

SHOCK AND VIBRATION ENVIRONMENT IN COMMERCIAL TRUCKS/TRAILER UNC 00315-001+ ENVIRONMENT IN VEHICLES TRAVELING OVER PAVED ROADS $\quad$ UNC $00317-001+$ VIBRATION-ACCELERATIONS IN HIGHWAY OPERAIION OF TRUCKS UNC 00370-001* SHOCK ZONES REGISTEREO INCARGO DURING SURFACE TRANSFER UNC 00813-001. DYNAMIC ENVIRONMENT ON A.FLAT BED TRACTOR/TRAILER (AEC /DOD) UNC 01006-008 FINAL TANALYSIS, AECIDOD FLATBED TRUC VIBRATION/SHOCK, $21 / 2$ TON TRUCK

VIBRATIONISHOCK AIR SUSPENSION VAN

RAIL HUMF SHOCK CRITERIA SUMMARY

BOXCAR SHOCK CRITERIA SUMMARY

ATMX CAR SHOCK CRITERIA SUMMARY

SHOCKIVIBRATION ENVIRONMENT SUMMARY, RAIL TRANSPORT

SHOCK SPECTRA SUMMARY, SIX-ATMX CAR TRAIN

SPEEDS, SWITCHING, OBSERVED AT VARIOUS HUMP YARDS

SPEEDS, SWITCHING, OBSERVATION OF, AT ILLINOIS CENTRAL RR YARDS

SPEEDS, SWITCHING, OBSERVED

SHOCK, SWITCHING, RAIL, OCCURANCE OF

SPEED, SWITCHING, DATA COMPARED WITH NEW DATA, NY CENTRAL RR

SPEED, HUMPING, FREQUENCY OF IMPACTS AT VARIOUS

RAIL SWITCHING AND HUMPING OPERATIONS

$\begin{array}{ll}\text { UNC } & \text { A1202-004 } \\ \text { UNC } & 81203-002 * \\ \text { UNC } & 81210-002+ \\ \text { UNC } & 81320-011+ \\ \text { UNC } & C 1215-012+ \\ \text { UNC } & 00284-001+ \\ \text { UNC } & 00285-004+ \\ \text { UNC } & 00286-001+ \\ \text { UNC } & 00287-001+ \\ \text { UNC } & 00288-001+ \\ \text { UNC } & 00289-001+ \\ \text { UNC } & 00290-001+\end{array}$




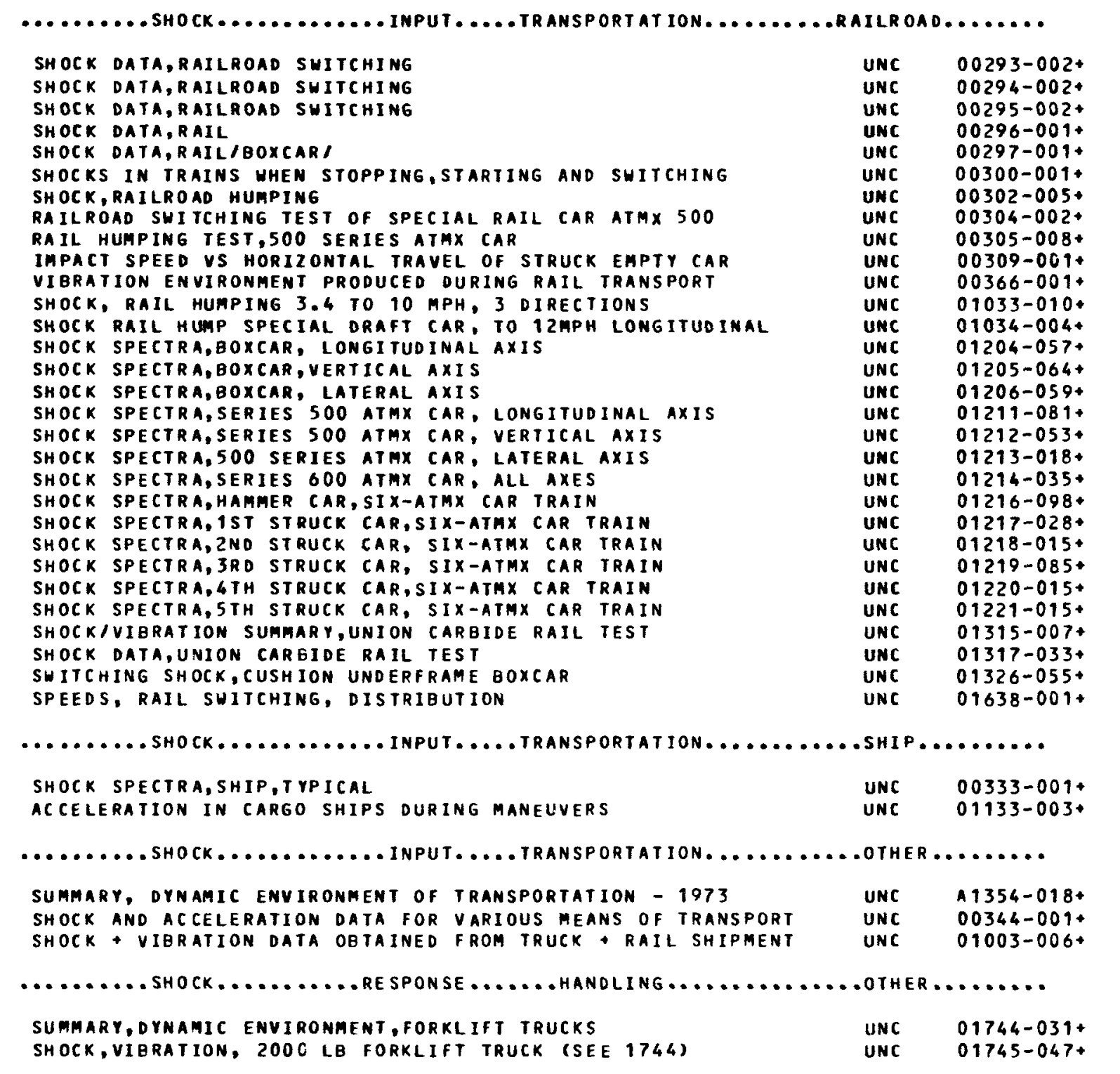




\begin{tabular}{|c|c|c|}
\hline $\begin{array}{l}\text { HOCK, RESPONSE, } 3000 \text { LB FORKLIFT TRUCK (SEE 1744) } \\
\text { HOCK, RESPONSE, } 4000 \text { B FORKLIFT TRUCK (SEE 1744) } \\
\text { HOCK, VIBRATION, } 7000 \text { LB FORKLIFT TRUCK (SEE 1744) }\end{array}$ & $\begin{array}{l}\text { UNC } \\
\text { UNC } \\
\text { UNC }\end{array}$ & $\begin{array}{l}01747-057 \\
01750-058+ \\
01752-041+\end{array}$ \\
\hline
\end{tabular}

......... SHOCK.......... RE SPONSE .... TRANSPORTAT1ON...............

$\begin{array}{llll}\text { SHOCK IVIBRATION SUMMARY,UNION CARBIDE TRUCK TEST } & \text { UNC } & 01312-007+ \\ \text { SHOCK DATA,UNION CARBIDE TRUCK TEST } & \text { UNC } & 01314-013+\end{array}$

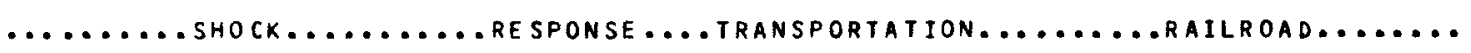

RAIL HUMP SHOCK CRITERIA SUMMARY

BOXCAR SHOCK CRITERIA SUMMARY

ATMX CAR SHOCK CRITERIA SUMMARY

SHOCKIVIBRATION ENVIRONMENT SUMMARY, RAIL TRANSPORT

SHOCK SPECTRA SUMMARY, SIX-ATMX CAR TRAIN

SHOCK SPECTRA, BOXCAR, LONGITUDINAL AXIS

SHOCK SPECTRA, BOXCAR, VERTICAL AXIS

SHOCK SPECTRA,BOXCAR, LATERAL AXIS

SHOCK SPECTRA, SERIES 500 ATMX CAR, LONGITUDINAL AXIS

SHOCK SPECTRA, SERIES 500 ATMX CAR, VERTICAL AXIS

SHOCK SPECTRA, 500 SERIES ATMX CAR, LATERAL AXIS

SHOCK SPECTRA, SERIES GOO ATMX CAR, ALL AXES

SHOCK SPECTRA, HAMMER CAR, SIX-ATMX CAR TRAIN

SHOCK SPECTRA, 1ST STRUCK CAR, SIX-ATMX CAR TRAIN

SHOCK SPECTRA, 2ND STRUCK CAR, SIX-ATMX CAR TRAIN

SHOCK SPECTRA, 3RD STRUCK CAR, SIX-ATMX CAR TRAIN

SHOCK SPECTRA, 4TH STRUCK CAR,SIX-ATMX CAR TRAIN

SHOCK SPECTRA, STH STRUCK CAR, SIX-ATMX CAR TRAIN

SHOCKIVIERATION SUMMARY, UNION CARBIDE RAIL TEST

SHOCK DATA, UNION CAREIDE RAIL TEST

SWITCHING SHOCK, CUSHION UNOERFRAME BOXCAR

$\begin{array}{ll}\text { UNC } & A 1202-004+ \\ \text { UNC } & 81203-002+ \\ \text { UNC } & 81210-002+ \\ \text { UNC } & 81320-011+ \\ \text { UNC } & 01215-012+ \\ \text { UNC } & 01204-057+ \\ \text { UNC } & 01205-064+ \\ \text { UNC } & 01206-059+ \\ \text { UNC } & 01211-081+ \\ \text { UNC } & 01212-053+ \\ \text { UNC } & 01213-018+ \\ \text { UNC } & 01214-035+ \\ \text { UNC } & 01216-098+ \\ \text { UNC } & 01217-028+ \\ \text { UNC } & 01218-015+ \\ \text { UNC } & 01219-085+ \\ \text { UNC } & 01220-015+ \\ \text { UNC } & 01221-015+ \\ \text { UNC } & 01315-007+ \\ \text { UNC } & 01317-033+ \\ \text { UNC } & 01326-055+\end{array}$

......... SHOCK.......... RESPONSE.... TRANSPORTATION.......... OTHER .......

SHOCK + VIERATION DATA OETAINED FROM TRUCK + RAIL SHIPMENT UNC 01003-006+

(IMPACT) .........SHOCK............ INPUT........GENERAL

OTHER.........

APPROXIMATE DURATION OF SOME SHORT ACCELERATION LOADS

UNC $\quad 00734-001$.

(IMPACT)

...........shock.............. InPUT

.HANDLING

OTHER..........

MAXIMUM DROPS, PACKAGES IN TRANSIT

UNC $\quad 01608-004+$ 
IEMPERATURE REGIME FOR DESERT REGIONS

TEMPERATURES, 1"-99' DESIGN,FOR UNITED STATES AND CANADA

ENVIRONMENT CONDITIONS PREVAILING DURING TRANSIT

TEMPERATURE, EXTREMES I DURATI ON, FOR STORAGE, HANDLING, TRANSPJRT

TE MPERATURE EXTREMES IN TRANSIT

RADIATION, MEAN DAILY TOTAL, FLAT SURFACE, LOCATIONS, USICANADA

UNC $\quad 00006-002+$

TYPHOON REPORT - PACIFIC OCEAN - 1959

TYPHOON REPORT - PACIFIC OCEAN - 1960

TYPHOON REPORT - PACIFIC OCEAN - 1961

TYPHOON REPORT - PACIFIC OCEAN - 1962

TYPHOON REPORT - PACIFIC OCEAN - 1963

TYPHOON REPORT - PACIFIC OCEAN - 1965

$\begin{array}{ll}\text { UNC } & 00018-002+ \\ \text { UNC } & 00078-001+ \\ \text { UNC } & 00080-002+ \\ \text { UNC } & 00084-001+ \\ \text { UNC } & 00495-004+ \\ \text { UNC } & 01802-003+ \\ \text { UNC } & 01803-003+ \\ \text { UNC } & 01804-003+ \\ \text { UNC } & 01805-004+ \\ \text { UNC } & 01806-003+ \\ \text { UNC } & 01807-003+\end{array}$

....... IEMPERATURE.

INPUT

IRANSPORTATION.

RCRAFT .

TE MPERATURES, CARGO COMPARTMENT, C119C AIRCRAFT, ALASKA, WINTER

TEMPERATURES, , IRCRAFT COMPARTMENT

TEMPERATURES, MAXIMUMIMINIMUM, IN AIR AND AIRCRAFT CARGO HOLOS

TE MPERATURE S, COMPARTMENT, C141A

FREQUENCY OF OCCURENCE OF AIRCRAFT ICING PARAMETERS

UNC $\quad 00061-001+$

TEMP/TIME DATA AIRCRAFT, TRUCK, TRAIN, ATMX

$\begin{array}{ll}\text { UNC } & 00061-001+ \\ \text { UNC } & 0006-001+ \\ \text { UNC } & 0006-001+ \\ \text { UNC } & 00066-001+ \\ \text { UNC } & 01089-002+\end{array}$

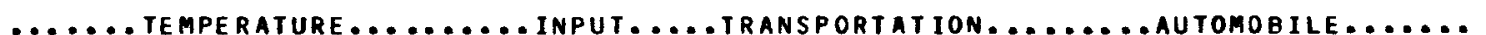

$\begin{array}{lll}\text { TEMPERATURE/TIME DATA,TRUCK (SEE 1288) } & \text { UNC } & 01309-004+ \\ \text { TEMPERATURE, TRUCK CARGO, HIGH AND LOH } & \text { UNC } & 01327-027 \text { * }\end{array}$

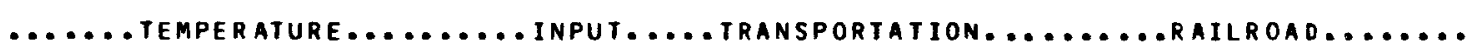

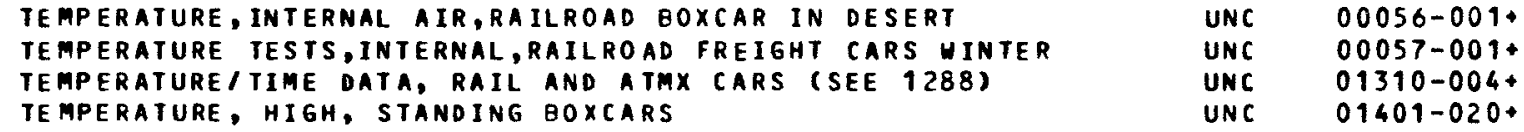

....... TEMPER

TEMPERATURES AND RELATIVE HUMIDITY,SHIP HOLD

UNC $00058-001+$ TE MPERATURES MAXIMUM AND MINIMUM, AT SEA

UNC $00059-001+$ HUMIDITY, REL ATIVE, ABOARO SHIP

UNC $\quad 00232-001+$

TEMPERATURE IN SHIPS HOLDS

UNC $\quad 01262-003$




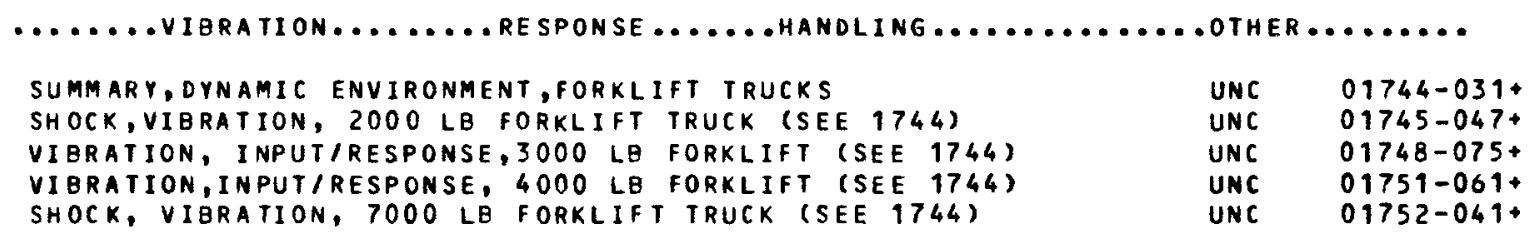

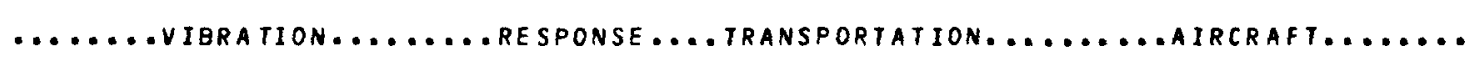

VIBRATION NC 135 AFT PASSENGER COMPARTMENT UNC 01318-010+

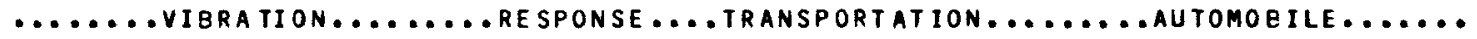

$\begin{array}{llll}\text { VIBRATION ENVIRONMENT, AEC VAN WITH TRANSIT RIG SHIPPING CONT } & \text { UNC } & 01245-061+ \\ \text { SHOCKIVIBRATION SUMMARY, UNION CARBIDE TRUCK TEST } & \text { UNC } & 01312-007+ \\ \text { VIBRATION DATA, UNION CARBIDE TRUCK TEST } & \text { UNC } & 01313-045+\end{array}$

VIBRATION DATA, UNION CARBIDE TRUCK TEST UNC 01313-045

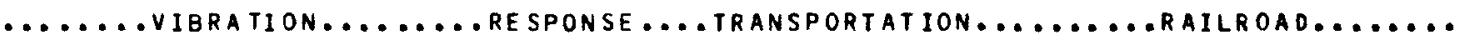

$\begin{array}{llll}\text { SHOCXIVIBRATION ENVIRONMENT SUMMARY, RAIL TRANSPORT } & \text { UNC } & \text { B 1320-011* } \\ \text { SHOCKIVIBRATION SUMMARY,UNION CARBIDE RAIL TEST } & \text { UNC } & 01315-007+\end{array}$

$\begin{array}{llll}\text { SHOCKIVIBRATION SUMMARY,UNION CARBIDE RAIL TEST } & \text { UNC } & 01315-007+ \\ \text { VIBRATION DATA,UNION CARBIDE RAIL TEST } & \text { UNC } & 01316-065+\end{array}$

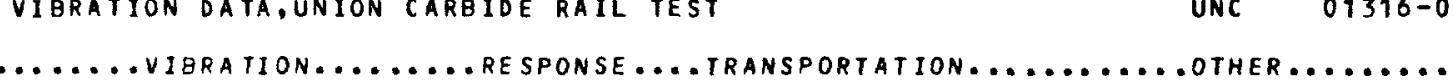

SHOCK + VIBRATION DATA OBTAINED FROM TRUCK + RAIL SHIPMENT UNC 01003-006 +

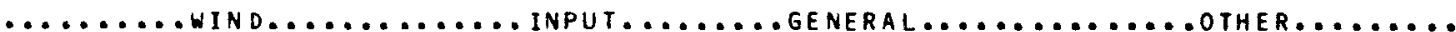

ENVIRONMENT CONDITIONS PREVAILING DURING TRANSIT

WIND VELOCITIES, WORLDWIDE, AND DISTRIBUTION IN TEMPERATE $20 N E$ UNC 00258-001+

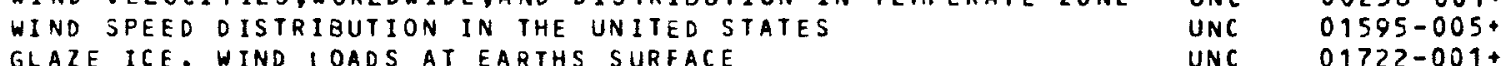

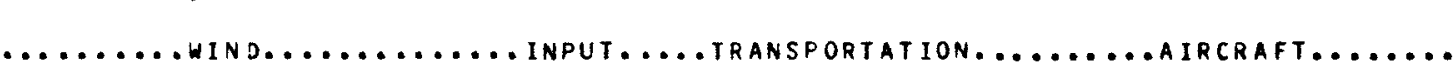

$\begin{array}{llll}\text { FATI IUE DAMAGE PARAMETERS, TRANSPORT AIRCRAFT } & \text { UNC } & 01085-001 * \\ \text { GUST LOAD FREQUENCY, AIRCRAFT FLIGHTS, 40-45KILOFEET, IN STORM UNC } & 01087-002 *\end{array}$

$\begin{array}{llll}\text { GUST LOAD FREQUENCY, AIRCRAFT FLIGHTS, 40-45KILOFEET, IN STORM } & \text { UNC } & 01087-002+ \\ \text { AIRCRAFT RECORDINGS DURING A TURBULENCE INCIDENT } & \text { UNC } & 01090-001+\end{array}$

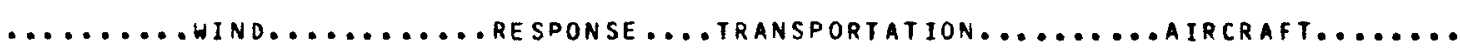

GUST LOAD FREQUENCY, AIRCRAFT FLIGHTS,40-45KILOFEET,IN STORM UNC 01087-002+

AIRCRAFT RECOROINGS DURING TURBULENCE INCIDENT UNC 01090-001+ 


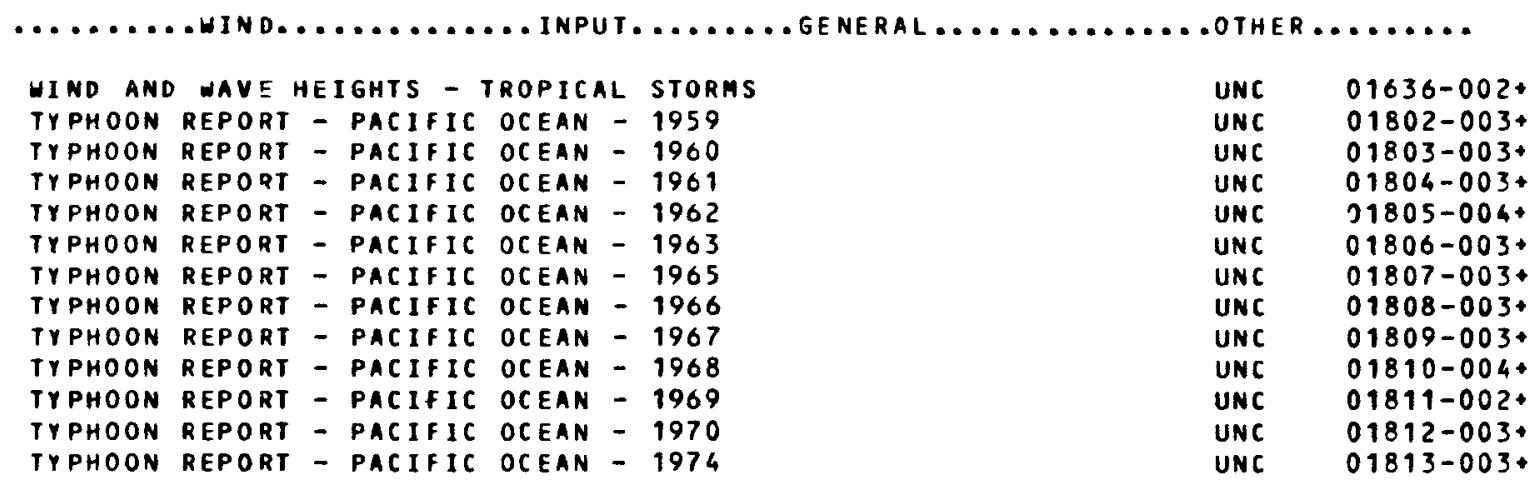


0

0 
Abnormal Environments 


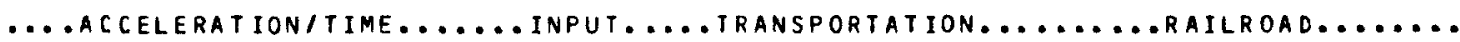

TRAIN ACCIDENT RATES, 1965-1974

UNC

$01920-004+$

(EARTH QUAKE)

... A ACCELERATION/TIME....... INPUT
EARTHQUAKES IN THE UNITED STATES
EARTHQUAKE, STRONG MOTION - ACCEL

EARTHQUAKE, STRONG MOTION - ACCELOGRAMS G VS TIME

EARTHQUAKE, STRONG MOTION - ACCELOGRAMS G VS TIME

EARTHQUAKE - ROCK MOTION ACCELOGRAMS G VS TIME, DISTANCE

.OTHER..........

STRONG MOTION EARTHQUAKE ACCELOGRAMS - PART F

STRONG MOTION EARTHQUAKE ACCELOGRAMS - PART $G$

STRONG MOTION EARTHQUAKE ACCELOGRAMS - PART H

STRONG MOTION EARTHQUAKE ACCELOGRAMS- VOL I,PT I, DATA

STRONG MOTION EARTHQUAKE ACCELOGRAMS- VOL I,PT J, DATA

STRONG MOTION EARTHQUAKE ACCELOGRAMS - VOL I,PT K, DATA

STRONG MOTION EARTHQUAKE ACCELOGRAMS-VOL III,RES. SPECTRA

SIRONG MOIION EARTHQUAKE ACCELOGRAMS-VOL IV,FOURIER SPECTRA

EARTHQUAKE, ANCHORAGE, 1964 S ENGINEERING INFORMATION

EARTHQUAKE ACCELOGRAMS-DIGITIZED AND PLOTTED DATA

EARTHQUAKE ACCELOGRAMS-RESPONSE SPECTRA

EARTHQUAKE ACCELOGRAMS-RESPONSE SPECTRA

EARTHQUAKE ACCELOGRAMS-FQURIER SPECTRA

UNC $00948-004$ +

UNC 01526-004*

UNC $\quad 01527-004+$

UNC $01528-001+$

UNC $01570-006+$

UNC $01579-004$ t

UNC $01572-004$ *

UNC $01572-004+$

UNC $\quad 01600-004$.

UNC $01601-0044$

UNC $\quad 01602-004^{+}$

UNC $\quad 01603-003+$

UNC $\quad 01604-002+$

UNC $01661-002$.

UNC $\quad 01661-002+$

UNC $01853-004$

UNC $01853-004+$

UNC $\quad 01855-003$.

( IMPAC T )

(IMPACT)

(IMPACT)
.... ACGELERAT IONITIME

APPROXIMATE DURATION OF SOME SHORT ACCELERATION LOADS

. OACCELERATION/TIME.0.

ANALYSIS OF DIRECIION OF LOAOS IN AIRCRAFT ACCIDENTS ACCELERATION OF FLOOR-AIRCRAFT CRASHES

AC CELERATION, AIRCRAFT CRASH-VARIOUS ANGLES OF IMPACT

FLOOR ACCELERATIONS, TRANSPORT AIRCRAFT ACCIDENTS

CIVIL AVIATION ACCIDENT DATA - 1962 TO 1972

AI RCRAFT ACCIDENT - EOEING $707-8 / 28 / 73$

AI RCRAFT ACCIDENT - DOEING $747-4 / 12172$

A RISK ANO COMPARATIVE ANALYSIS OF NAVY AIRCRAFT DATA

UNC $00734-001+$

... A ACELERATION/TIME....... INPUT.... TKANSPORTation.

AC CELERATIONS, AUTOMOBILE CRASH (HEAD-ON)

DECELERATION PATTERNS FOR AUTOMOBILE HEAD-ON COLLISIONS

TRUCK ACCIDENTS, NHTSA, FINAL REPORT

TRUCK ACCIDENTS, NHTSA, VOLG, NO. 4

TRUCK ACCIDENTS, NHTSA, VOLS, NO.

TRUCK ACCIDENTS, NHTSA, VOL $6, N O . O$

TRUCK ACCIDENTS, NHTSA, VOLG, NO.?

IRCRAFT.........

$\begin{array}{ll}\text { UNC } & 00751-001+ \\ \text { UNC } & 00752-001+ \\ \text { UNC } & 00753-008+ \\ \text { UNC } & 01629-001+ \\ \text { UNC } & 01664-009+ \\ \text { UNC } & 01801-001+ \\ \text { UNC } & 01818-001+ \\ \text { UNC } & 01857-001+\end{array}$


(IMPACT)

(I MPACT)

(IMPACT)

(PUNC TURE)

(PUNCTURE)

(PUNC TURE)

(PUNCTURE)
TRUCK ACCIDENTS, NHTSA, VOL G, NO.8

TRUCK ACCIDENTS, NHTSA, VOL 6, NO, 9

CAR ACCIDENT, MHTSA, VOL.6, MO. 11

TRUCK ACCIDENT, MHTSA, VOL.6, NO.10

TRUCK ACCIOENT, NHTSA, VOL.6, NO.10

TRUCKIPICKUP TRUCK WICAMPER AND TRAILER COLLISION
UNC $01880-011$.
UNC $01898-005$.
UNC $01899-005$.
UNC $01900-004$

UNC

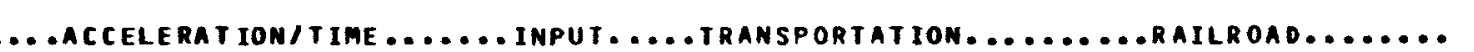

RAIL ACCIDENT STATISTICS

UNC 01020-025+

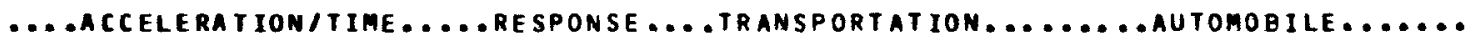

TRUCK ACCIDENTS, NHTSA, FINAL REPORT

TRUCK ACCIDE TTS MHTSA VOLG, NO

TRUCX AC CIDENTS, NHTSA, VOLG, NO. 5

TRUC K ACCIDENTS, NHTSA, VOL 6, NO.6

TRUCX ACCIDENTS, NHTSA, VOL 6, NO.7

TRUCK ACC IDENTS, NHTSA, VOL 6, NO.8

TRUCK ACCIDENTS NHTSA, VOL6, NO. 9

CAR ACCIDENT. NHTSA, VOL.6, NO.11

TRUCK ACCIDE MT, NHTSA, VOL.6. NO.10

TRUCK ACCIDENT, NHTSA, VOL.6, NO.10

TRUCK ACCIDENT, NHTSA, VOL

UNC 01875-019.

UNC $01876-003+$

UNC $\quad 01877-004+$

UNC $01878-008$ *

UNC $01879-006+$

UNC 01880-011+

UNC 01881-014t

UNC $\quad 01898-005$.

UNC $01899-005$.

UNC $01899-005$

UNC

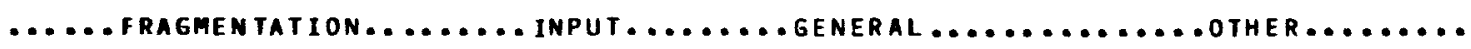

FRAGMENTS, MASS, VELOCITY, STANDARD ARTILLERY SHELLS UNC 01814-002,

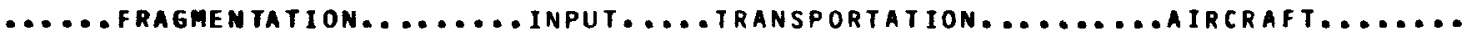

ROTOR FRAGMENT CONTAINMENT-JET ENGINE FAILURE - 1972 UNC 01781-010+

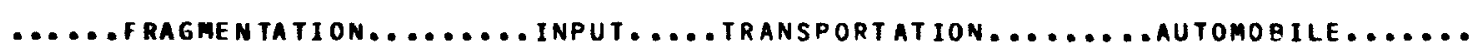

MOTOR CARRIER ACCIDENT ENVIRONMENT - TAC STUDY

TRUCK ACCIDENT-FLORIOA-8/8/71

TRUCK ACCIDENT REPORTS, NHTSA, NO. 7

TRUCK ACCIDENT REPORTS, NHTSA, NO. 8

TRUCK ACCIDENT REPORTS, NHTSA, NO.

$\begin{array}{ll}\text { UNC } & 11662-007+ \\ \text { UNC } & 01695-003+ \\ \text { UNC } & 01753-005+ \\ \text { UNC } & 01754-004+ \\ \text { UNC } & 01755-005+\end{array}$

..... . FRAGMENTATION......... INPUT . ... TRANSPORTATION.

RAILROAD ACCIDENT ENVIRONMENT - TAC STUDY

RAIL ACCIDENT, HOUSTON,TEXAS 21 SEPT, 1974

UNC $1563-006+$

UNC $\quad 01893-014+$ 
(PUNCTURE)

(PUNCTURE)

(EXPLOSION)
......FRAGMENTATION......... INPUT ..... TRANSPORTATION .

IMPACT, PENETRATION, SHIP COLLISION AND GROUNDING

$01550-001+$

CARGO RESPONSE TRUCK AND RAIL ACCIOENTS UNC 01688-002* TRUCK ACCIDENT-FLORIDA-818/71 UNC 01695-003*

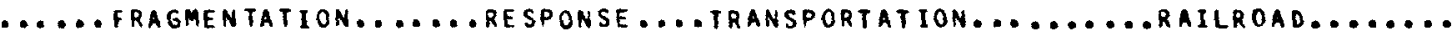

CARGO RESPONSE TRUCK AND RAIL ACCCIDENTS SEE EDB1688

UNC $\quad 01699-002+$

RA TL ACCIDENT, HOUSTON,TEXAS, T9OCTI9?1

$\begin{array}{lll}\text { RAIL ACCIDENT, CRESCENT CITY, 19AUG1970 } & \text { UNC } & 01847-009+ \\ \text { RAIL ACCIDENT, HOUSTON,TEXAS } 29 \text { SEPT, } 1974 & \text { UNC } & 01893-014+\end{array}$

$01846-006+$

..... fragmentation......... input.....transportation......... automobile......

TRUCK ACCIDENT-NEW JERSEY $9 / 21 / 72$

TRUCK ACCIDENT-GEORGIA-6/4/71

UNC $\quad 01690-003+$

TRUCK ACCIDENT-VIRGINIA-3/9172

$01694-003+$

$01696-003+$

\begin{tabular}{|c|c|c|}
\hline SUMMARY, OYNAMIC ENVI RONMENT, FORKLIFT TRUCXS & UNC & $01744-0314$ \\
\hline $\begin{array}{l}\text { SHOCK, VIBRATION, } 2000 \text { LB FORKLIFT TRUCK (SEE 1744) } \\
\text { VIBRATION, INPUT/RESPONSE, } 3000 \text { LB FORKLIFT (SEE 1744) } \\
\text { VIBRATION,INPUTIRESPONSE, } 4000 \text { LE FORKLIFT (SEE 1744) } \\
\text { SHOCK, VIBRATION, 70OO LB FORKLIFT TRUCK (SEE 1744) }\end{array}$ & $\begin{array}{l}\text { UNC } \\
\text { UNC } \\
\text { UNC } \\
\text { UNC }\end{array}$ & $\begin{array}{l}01745-047+ \\
01748-075+ \\
01751-061+ \\
01752-041^{+}\end{array}$ \\
\hline
\end{tabular}




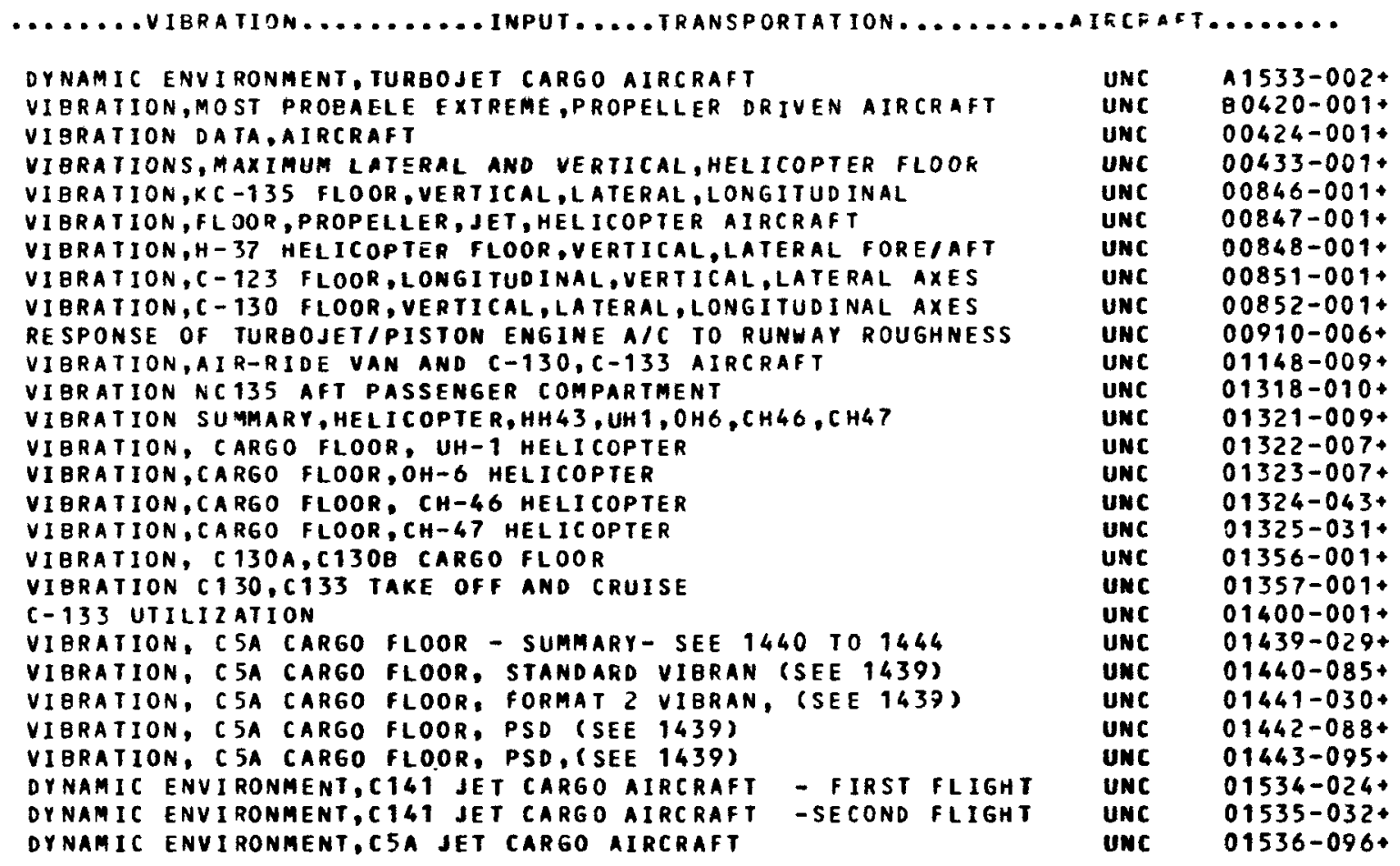

........VIERATION........... INPUT..... TRANSPORTATION.........AUTOMOEILE......

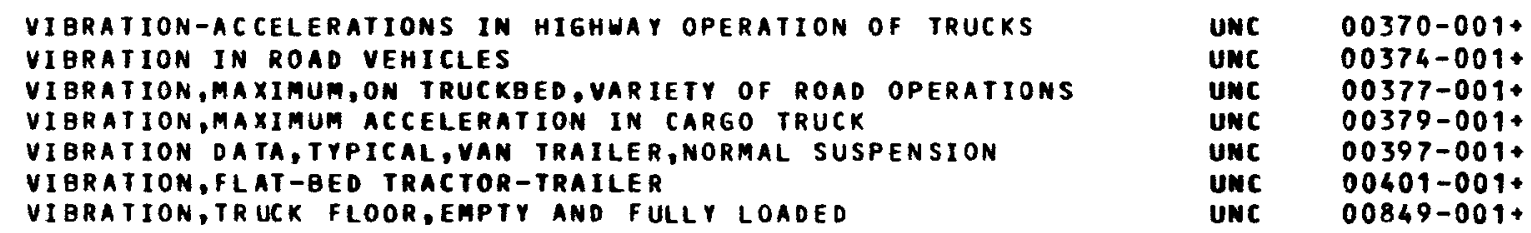

OYNAMIC ENVIRONMENT ON A FLAT BED TRACTOR/TRAILER (AEC/DOD) FINAL ANALYSIS, AECIDOD FLATBED TRUCK TEST

VIBRATION ENUIRONAEMT, AEC VAN WITH TRANSIT RIG SHIPPING CONT

VIBRATION ENUIRONMENT, AEC VAN WI

VIBRATION/SHOCK AIR SUSPENSION VAN

SH OCK /VIERATION SUMMARY, UNION CARBIDE TRUCK TEST

VIBRATION DATA, UNION CARBIOE TRUCK TEST

VIBRATION, TRUCK, TANDEM AXLE, AIR BAG SUSPENSION

VIBRATION IN COMMON MOTOR CARRIERS

$\begin{array}{ll}\text { UNC } & 01006-008+ \\ \text { UNC } & 01083-041+ \\ \text { UNC } & 01245-061+ \\ \text { UNC } & 01264-008+ \\ \text { UNC } & 01277-006+ \\ \text { UNC } & 01312-007+ \\ \text { UNC } & 01313-045+ \\ \text { UNC } & 01361-001+ \\ \text { UNC } & 01742-007+\end{array}$


SHOCK/VIERATION ENVIRONMENT SUMMARY, RAIL TRANSPORT VIBRATION,RAIL TRANSPORT, SUMMARY OF DATA ON

VIBRAIION DATA,RAIL

VIBRATION TESTS, RAILROAD, SPEED $40 \mathrm{MPH}$

VIBRATION TESTS, RAILROAD, SPEED $73 \mathrm{MPH}$

VIBRATION TESTS, RAILROAD, SPEED $80 \mathrm{MPH}$

VIBRATIONS IN RAILROAD FREIGHT CARS AT SPEEDS FROM 31-73MPH

VIBRATIONS MEASURED ON ATMX 5OO CAR AT SPEEDS 30-7OMPH

VIBRATION ENVIRONMENT PRODUCEO DURING RAIL TRANSPORT

VIBRATION,RAILROAD, SOFT SPRUNG CAR, VERTICAL AND LATERAL

VIBRATION, RAILROAD CONTINUOUS AND TRANSIENT

VIBRATION, RA ILROAD ENVELOPES OIRECTIONAL COMPOSITES

SHOCKIVIBRATION SUMMARY, UNION CARBIDE RAIL TEST

VIBRATION DATA, UNION CARBIDE RAIL TEST
UNC B1320-011+

UNC 00355-001+

UNC 00357-001+

UNC 00358-001.

UNC 00350-001.

$00360-001$.

$00360-001$

$00362-001$

$00363-001+$

$00366-001+$

$00850-001$.

$01031-001+$

$01032-001+$

$01315-007+$

$01316-065$

........VIBRATION.......... INPUT ..... TRANSPORTATION..........SHIP........

VIBRATION,MOST EXTREME PROBABLE, EXPECTED IN SEA TRANSPORT VIBRATION MEASUREMENTS ON SHIPBOARD DURING TRANSPORT VIBRAIION DATA, SHIPS

VIBRATION DATA, SHIPS IN STRAIGHT RUNS AT VARIOUS SPEEDS VIBRATION, SHIPBOARD, NORMAL STEAMING AND HARD TURNS

VIBRATION DATA, SHIPS, HIGH SPEED MANEUVER AND CRASH BACK

VIBRATIONS, SHIPBOARD, FROM VARIOUS SOURCES

VIBRATIONS, MERCHANT SHIP SS WOLVERINE STATE

$80404-001+$

UNC $00202-001+$

$00405-001+$

$00407-001$.

$00407-001+$

$00408-001+$

$00409-001+$

$00415-001+$

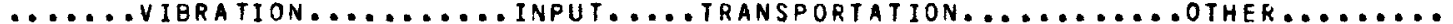

SUMMAFY, DYNAMIC ENVIRONEENT OF TQANSPORTATION - 1973

YIERATION SUMMARIES OF EDB 1354 IN PSD FORMATS

VISRATION-MAXIMUM ACCELERATIONS FOR VARIOUS VEHICLES

SHOCK + VIBRATION DATA OBTAINED FROM TRUCK + RAIL SHIPMENT

UNC
UNC

A $1354-018$

TRANSPORTATION VIBRATION ENVIRONMENTS

B1354-006+

$00368-001$

$01003-006$

$01176-002+$

\begin{tabular}{|c|c|c|c|c|c|c|}
\hline TYPHOON & REPORT & $-P A C I F I C$ & OCEAN & -1959 & UNC & $01802-003+$ \\
\hline TYPHOON & REPORT & - PACIFIC & OCEAN & -1960 & UNC & $01803-003+$ \\
\hline TYPHOON & REPORT & - PACIFIC & OCEAN & -1961 & UNC & $01804-003+$ \\
\hline $\begin{array}{l}\text { TYPHOON } \\
\text { TYPHOON }\end{array}$ & $\begin{array}{l}\text { REPORT } \\
\text { REPORT }\end{array}$ & $\begin{array}{l}\text { - PACIFIC } \\
\text { - PACIFIC }\end{array}$ & $\begin{array}{l}\text { OCEAN } \\
\text { OCEAN }\end{array}$ & $\begin{array}{l}-1962 \\
-1903\end{array}$ & $\begin{array}{l}\text { UNC } \\
\text { UNC }\end{array}$ & $\begin{array}{l}01805-004+ \\
01806-003+\end{array}$ \\
\hline TYPHOON & REPORT & - PACIFIC & OCEAN & $\begin{array}{l}-1965 \\
-1906\end{array}$ & UNC & $01807-003+$ \\
\hline $\begin{array}{l}\text { TYPHOON } \\
\text { TYPHOON }\end{array}$ & $\begin{array}{l}\text { REPORT } \\
\text { REPORT }\end{array}$ & $\begin{array}{l}\text { - PACIFIC } \\
\text { - PACIFIC }\end{array}$ & $\begin{array}{l}\text { OCEAN } \\
\text { OCEAN }\end{array}$ & $\begin{array}{l}-1906 \\
-\quad 1967\end{array}$ & UNC & $\begin{array}{l}01808-003+ \\
01809-003+\end{array}$ \\
\hline TYPHOON & REPORT & - PACIFIC & OCEAN & -1968 & UNC & $01810-004+$ \\
\hline TYPHOON & REPORT & - PACIFIC & OCEAN & -1969 & UNC & $01811-002+$ \\
\hline TYPHOON & REPORT & - PACIFIC & OCEAN & -1974 & UNC & $01813-003$ \\
\hline TROP ICAL & $L C Y C L O$ & VES & & & UNC & $01856-002+$ \\
\hline
\end{tabular}


( CRUSH)

(I MHERSI ON)

(EXPLOSION)

(EXPLOSION)

(EXPLOSION)

(LIGHTNING)

(LIGHTNING)

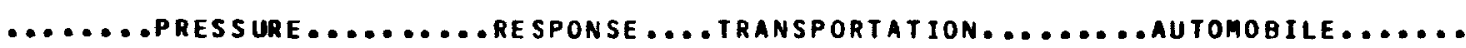

CARGO RESPONSE TRUCK AND RAIL ACCIDENTS

UNC $\quad 01688-002+$

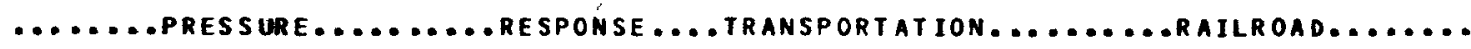

CARGO RESPONSE TRUCK AND RAIL ACCCIDENTS SEE EDB1688

UNC $01699-002+$

-.........PRESSURE

. INPUT..... . TRANSPORTATION.

A/C ACCIDENT DEHAVILLAND DHC 16 FEB 1970

A/C ACCIDENT DCS MAY 1970

A/C ACCIDENT SKYYAN SERIES 3 JULY 1970

UNC 01679-003

UNC 01682-003

UNC 01683-003.

........PRESS URE ........... INPUT..... TRANSPORTATION ........AUTOMOBILE......

HYDROGEN TRANSPORT AND HANDLING EXPLOSION UNC 01758-001+

........PRESSURE............ INPUT ..... TRANSPORTATION.........RAILR OAD.......

RAIL ACCIDENT, EAST ST LOUIS, ILLINOIS - $1 / 22 / 72$

RA IL ACCIDENT, CRETE, NEBRASKA - 2118169

RAIL ACCIDENT, HOUSTON, TEXAS - $10 / 19171$

RAIL ACCIDENT, DECATUR, ILL 19 JULY, 1974

RAIL ACCIDENT, BENSON,AR 24 MAY, 1973

RAIL ACCIDENT, HOUSTON, TEXAS 21 SEPT, 1974

UNC $\quad 01706-003+$

UNC $\quad 01707-003$

UNC $01708-001$.

UNC $\quad 01708-001$.

UNC $01891-016$

UNC $01893-014$.

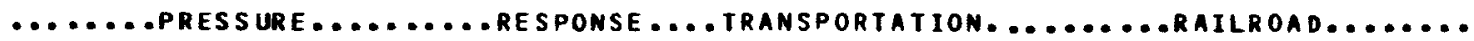

RAIL ACCIDENT, DECATUR, ILL 19 JULY, 1974

RAIL ACCIDENI,BENSON,AR 24 MAY, 1973

RAIL ACCIDENT, HOUSTON, TEXAS 21 SEPT, 1974

UNC 01889-012

UNC 01891-016

UNC $01893-014+$

OTHER .........

VOLTAGE AND CURRENT CHARACTERISTICS OF LIGHTNING STRIKE UNC 00464-002* PROPERTIES OF LIGHTNING STROKES

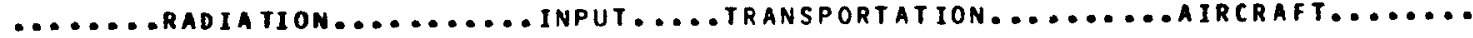

LIGHTNING THREAT TO AIRCRAFT

UNC $\quad 01000-002+$ LI GHTNING HAZARD TO AIRCRAFT 
MOTOR CARRIER ACCIDENT ENVIRONMENT - TAC STUDY

TRUCK ACCIDENT REPORTS, NHTSA, NO.?

TRUCK ACCIDENT REPORTS, NHTSA, NO. 8

TRUCK ACCIDENT REPORTS, NHTSA, NO. 9

TRUCK ACCIDENT, NHTSA, VOL.6, NO.10

TRUCK ACCIDENT, NHTSA, VOL.6, NO.1

IRUCKIPICKUP TRUCK HICAMPER AND TRAILER COLLISION

$\begin{array}{ll}\text { UNC } & \text { A1662-007+ } \\ \text { UNC } & 01753-005+ \\ \text { UNC } & 01754-004 \text { + } \\ \text { UNC } & 01755-005+ \\ \text { UNC } & 01899-005+ \\ \text { UNC } & 01900-004+ \\ \text { UNC } & 01001-015+\end{array}$

UNC 01901-015+
( $(R \cup S H)$

( $($ RUSH)

( $C R \cup S H)$

(IMPACT)

(IMPAC T)

(IMPACT) $\begin{array}{lll}\text { RAILROAD ACCIDENT ENVIRONMENT - TAC STUDY } & \text { UNC } & \text { A1603-006* } \\ \text { RAIL ACCIDENT, HOUSTON,TEXAS } 21 \text { SEPT, 1974 } & \text { UNC } & 01893-014 *\end{array}$

TRUCK ACCIDENT, NHTSA, VOL.6, NO.10

$\begin{array}{llll}\text { TRUCK ACCIDENT, NHTSA, VOL.6, NO.10 } & \text { UNC } & 01899-005+ \\ \text { TRUCK ACCIDENT, NHTSA, VOL.6, NO.12 } & \text { UNC } & 01900-004+\end{array}$

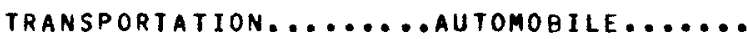

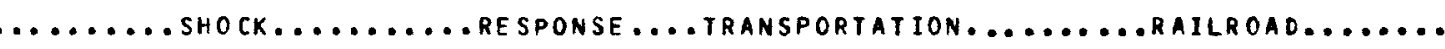

RAIL ACCIOENT,HOUSTON, TEXAS, 190CT1971 UNC 01846-006+

RAIL ACCIDENT, CRESCENT CITY, 19AUG1970 UNC 01847-009+

RAIL ACCIDENT, HOUSTON, TEXAS 21 SEPT, 1974 UNC 01893-014*

......... SHOCK........... INPUT ........ GENERAL............

APPROXIMATE OURATION OF SOME SHORT ACCELERATION LOADS UNC 00734-001+

......... SHOCK........... InPUT........HANDLING ........... OTHER.......

IMPACTS DUE TO LARGE DROPS WHEN LOADING UNC 00342-001+ MAXIMUM OROPS, PACKAGES IN TRANSIT UNC 01608-004+

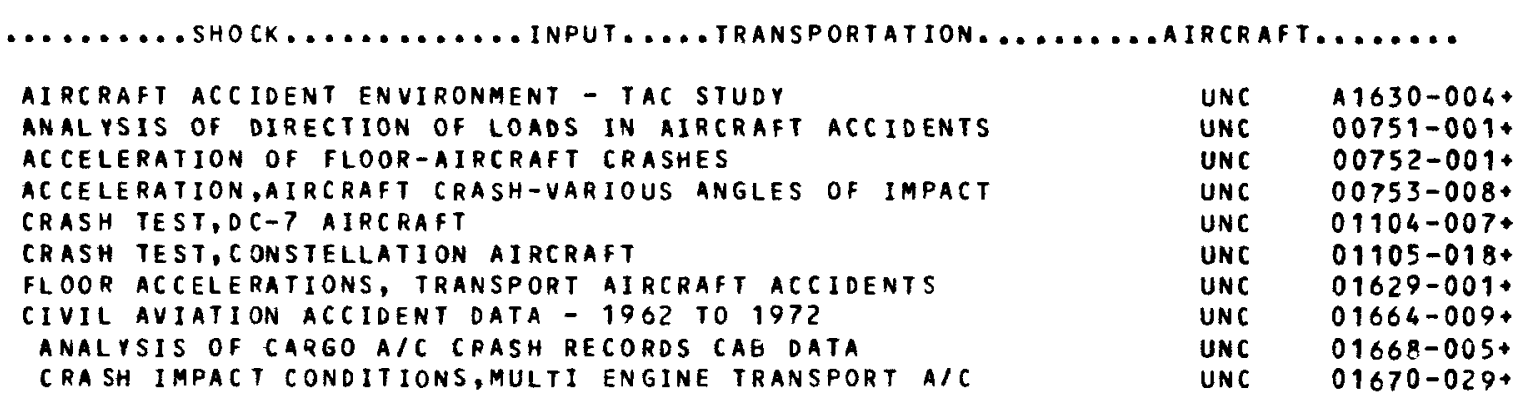


SURVEY OF AIC CRASH SURVIVABILITY, CARGO AT A/C CG

AC CI DENT DATA, MULTIENGINE AIRCRAFT

A/C ACCIDENT DCZ 62 SEPT 1970

AIC ACCIDENT MARTIN 404 OCT 1970

A/C ACCIDENT CONVAIR 580 DEC 1968

A/C ACCIDENT DEHAVILLAND DHC 16 FEB 1970

AIC ACCIDENT DOUGLAS C54D APRIL 1970

$A / C$ ACCIDENT DC 9 JAN 1970

AIC ACCIDENT DCQ MAY 1970

A/C ACCIDENT SKYVAN SERIES 3 JULY 1970

A/C ACCIDENT DC9 32 SEPT 1970

A/C ACCIDENT DC8 63F SEPT 1970

AIRCRAFT ACC IDENT REPORTS - NTSB - 1972

AIRCRAFT ACCIDENTS, IYPE, RATE, NTSB 1970

AIRCRAFT ACCIDENTS, TYPE, RATE, NTSB 1973

AIRCRAFT ACCIDENT - BOEING 707 - 1/16/74

AIRCRAFT CRASH ENVIRONMENTS - NAVY

AIRCRAFT ACCIDENT - CONVAIR 600-9127/73

AIRCRAFI DITCHING INVESTIGATION

A/C CRASH,B7D7, BOSTON, 3 NOY 1973

A RISK AND COMPARATIVE ANALYSIS OF NAUY AIRCRAFT DATA
AIRCRAFT MIDAIR COLLISIONS

$\begin{array}{ll}\text { UNC } & 01671-004+ \\ \text { UNC } & 01672-010+ \\ \text { UNC } & 01676-003+ \\ \text { UNC } & 01677-003+ \\ \text { UNC } & 01678-003+ \\ \text { UNC } & 01679-003+ \\ \text { UNC } & 01680-003+ \\ \text { UNC } & 01681-003+ \\ \text { UNC } & 01682-003+ \\ \text { UNC } & 01683-003+ \\ \text { UNC } & 01684-003+ \\ \text { UNC } & 01686-003+ \\ \text { UNC } & 01724-002+ \\ \text { UNC } & 01756-002+ \\ \text { UNC } & 01757-001+ \\ \text { UNC } & 01800-001+ \\ \text { UNC } & 01819-001+ \\ \text { UNC } & 01820-001+ \\ \text { UNC } & 01822-002+ \\ \text { UNC } & 01823-002+ \\ \text { UNC } & 01843-023+ \\ \text { UNC } & 01857-001+\end{array}$

(IMPACT)

AC CELERATIONS, AUTOMOBILE CRASH (HEAD-ON)

DECELERATION PATTERNS FOR AUTOMOBILE HEAD-ON COLLISIONS

AC CI DENT REPORT, TRUCK - VENTURA, CALIFORNIA, 8-18-71

TRUCK ACCIDENT SPEEDS-A SUMMARY

TRUCK ACCIDENT-NEW JERSEY $9121 / 72$

TRUCK ACCIDENT-ARIZONA $8 / 31170$

TRUCK ACCIDENT-VIRGINIA $4 / 13 / 72$

TRUCK ACCIDENT-PENN-9/5/71

TRUCK ACCIDENT-GEORGIA-6/4/71

TRUCK ACCIDENT-FLORIOA-8/8/71

TRUCK ACCIDENT-VIRGINIA- $19 / 72$

TRUCK ACCIDENT-TENN-5/13/72

STATISTICAL STUDY OF TRUCK ACCIDENT SPEEDS

RAIL/ROAD VEHICLE ACCIDENT, OKLAHOMA - 4/5/71-SEE 1710

RAIL/ROAD VEHICLE ACCIDENT, NEW YORK $-3 / 24 / 72$

RAIL/ROAD VEHICLE ACCIDENT, ILLINOIS -1/24/70-SEE 1714

TRUCK ACCIDENT REPORTS, NHTSA, NO.?

TRUCK ACCIDENT REPORTS, NHTSA, NO. 8

TRUCK ACCIDENT REPORTS, NHTSA, NO. 9

TRUCK ACCIDENT REPORTS - BOSTON UNIV., 1973

TRUCK ACCIDENT REPORTS - NHTSA, NO. 3

\section{TOHOB ILE........}

UNC 00738-0014

UNC 01659-003.

UNC 01687-002+

UNC $01690-003+$

UNC $01691-003$.

UNC $01692-003$.

UNC $01693-003$.

UNC $01694-003$

UNC 01695-003

UNC 01697-002

UNC 01698-012.

UNC 01719-001.

UNC $01712-003$

UNC 01715-001.

UNC 01754-004.

UNC 01755-005.

UNC 01770-004

UNC 01771-0034
UNC 01696-003.

UNC 01711-001.

UNC 01753-005 

. INPUT..... TRANSPORTATION...........AUTOMOBILE...

RA IL-HIGHWAY GRADE CROSSING ACCIDENTS, 1972 (SEE 1775) RAIL-HIGHWAY GRADE CROSSING ACCIDENTS, 1973 (SEE 1777)

TRUCK ACCIDENT - TENNESSEE - $8 / 27 / 73$

TRUCK ACCIDENT - VIRGINIA - 4/13/72

TRUCK ACCIDENT - TEXAS - 3/7/73

TRUCK ACCIDENT - IOWA - $4 / 3173$

TRUCK ACCIDENT - VIRGINIA - 3/28/74

TRUCK ACCIDENT - MASSACHUSETTS - $10 / 18173$

TRUCK/GUS ACCIDENT, NEW JERSEY, 19 OCT 1973

TRUCK ACCIDENTS, NHTSA, FINAL REPORT

TRUCK ACCIDENTS, NHTSA, VOLG, NO.G

TRUCK ACCIDENTS, NHTSA, VOL 6, NO.

TRUCK ACCIDENTS, NHTSA, VOLG, NO.

TRUCK ACCIDENTS, NHTSA, VOL6, NO.7

TRUCK ACCIOENTS, NHTSA, VOLG, NO.8

TRUCK ACCIDENTS, NHTSA, VOLO, NO

ACCI OENTS CANADA RAY CONTAINERS
$01776-001+$ $01778-001+$ $01783-001$. $01784-002+$ $01785-002$. $01786-001+$ $01787-003+$ $01788-002+$ $01845-014+$ $01845-0144$ $01875-019+$ $01876-003+$ $01877-004 *$ $01878-008+$ $01879-006+$ $01880-019+$ $01881-014+$ $01888-013+$

(IMPACT)
RAILROAD ACCIDENT ENVIRONMENT - TAC STUDY

RAIL ACCIDENT STATISTICS

COLLISIONS, RAIL, HEAD ON AND REAR END

RAIL ACCIDENT STATISTICS, 1969

RAIL ACCIOENT STATISTICS, 1971

ACCIDENT DATA RAILROAD 1952 THRU 1955

RAIL ACCIDEVT TANK CAR PUPTURE FIRE

RAIL ACCIDEVT TANK CAR RUPTURE,

OF RAM 1948-1965 SEE EDB 1689

RAIL ACCIDENT, COTULLA, TEXAS - 12/1173

RAIL ACCIDENT, INDIO, CALIFORNIA - 0/25/73

RAIL ACCIDENT, SALEM, ILLINOIS - 6/10/71

RAIL ACCIDENT, MAQUIN, ILLINOIS - $5 / 24 / 72$

RAIL ACCIDENT, TAFT, LOUISIANA- $2 / 21 / 73$

RAIL ACCIDENT, EAST ST LOUIS, ILLINOIS - $1 / 22172$

RAIL ACCIDENT, CRETE, NEBRASKA - 2118169

RAIL ACCIDENT, HOUSTON, TEXAS - $10 / 19171$

RAIL ACCIDENT, HERNDON, PENNSYLVANIA-3112172

RAIL IROAD VEHICLE ACCIDENT, OKLAHOMA - 4/5171

RAILIROAD VEHICLE ACCIOENT, NEW YORK -3/24/72-SEE 1712

RAIL/ROAD VEHICLE ACCIDENT, ILLINOIS $-1 / 24 / 70$

FREIGHT TRAIN ACCIDENTS, NUMBER OF CARS INVOLVED

RAIL -HIGHWAY GRADE CROSSING ACCIDENTS, 1972

RAIL-HIGHWAY GRADE CROSSING ACCIDENTS, 1973

RAIL ACCIDENT,ONEONTA, NY 12 FEB, 1976

RAIL ACCIDENT,MUSTANG, OK, ISEPT, 1974

$\begin{array}{ll}\text { UNC } & 11663-006 * \\ \text { UNC } & 01020-025+ \\ \text { UNC } & 01665-006+ \\ \text { UNC } & 01666-002+ \\ \text { UNC } & 01667-002+ \\ \text { UNC } & 01673-001+ \\ \text { UNC } & 01675-036+ \\ \text { UNC } & 01700-011+ \\ \text { UNC } & 01701-001+ \\ \text { UNC } & 01702-001+ \\ \text { UNC } & 01703-002+ \\ \text { UNC } & 01704-001+ \\ \text { UNC } & 01705-006+ \\ \text { UNC } & 01706-003+ \\ \text { UNC } & 01707-003+ \\ \text { UNC } & 01708-001+ \\ \text { UNC } & 01709-003+ \\ \text { UNC } & 01710-002 * \\ \text { UNC } & 01713-001+ \\ \text { UNC } & 01714-002+ \\ \text { UNC } & 01729-007 * \\ \text { UNC } & 01775-002+ \\ \text { UNC } & 01777-002+ \\ \text { UNC } & 01890-012+ \\ \text { UNC } & 01892-010 *\end{array}$


(IMPACI)

( IMP AC T )

(IMPAC T)

(IMPACT)

(IMPACT)

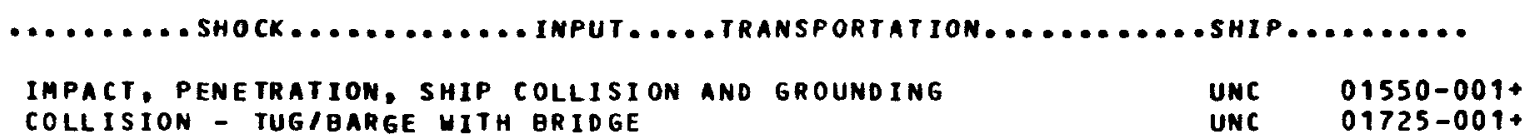

COLLISION - TUGIBARGE UITH BRIDGE

OTHER ............

MAXIMUM DROPS, PACKAGES IN TRANSIT

UNC $\quad 01608-004+$

SHOCK SPECTRA, STAGED A/C CRASH TESTS

A/C CRASH,B707,BOSTON, 3 NOV 1973 UNC 01843-023+

......... SHOCK........... RE SPONSE .... TRANSPORTATION........AUTOMOBILE......

CARGO RESPONSE TRUCK AND RAIL ACCIDENTS

TRANSPORT ACCIDENTS OF RAM 1948 TO 1965-UNITED KINGDOM

TRUCK ACCIDENT-FLORIDA-8/8/71

TRUCK ACCIDENTS, NHTSA, FINAL REPORT

TRUCK ACCIDENTS, NHTSA, YOLO NO 4

TRUCK ACCIDEMTS, MHTSA, VOL, NO. 4

,

TRUCK ACCIDENTS, NHTSA, VOL 6, NO.6

TRUCK

$6 . N 0.8$

TRUCK ACCIDENTS, NHTSA, VOL6, NO.9

AC CI DENTS, CANADA, RAM CONTAINERS

$\begin{array}{ll}\text { UNC } & 01688-002+ \\ \text { UNC } & 01689-011+ \\ \text { UNC } & 01695-003+ \\ \text { UNC } & 01875-019+ \\ \text { UNC } & 01876-003+ \\ \text { UNC } & 01877-004+ \\ \text { UNC } & 01878-008+ \\ \text { UNC } & 01879-006+ \\ \text { UNC } & 01880-011+ \\ \text { UNC } & 01881-014+ \\ \text { UNC } & 01888-013+\end{array}$

AILROAD........

CARGO RESPONSE TRUCK AND RAIL ACCCIDENTS SEE EDB1688

RAIL ACCIDENT, ONEONTA, NY 12 FEB, 1974

UNC 01699-002*

RAIL ACCIDENT, MUSTANG, OK, ISEPT, 1974

UNC $\quad 01890-012+$

UNC $01892-010$ + 
TYPHOON REPORT - PACIFIC OCEAN - 1959 TYPHOON REPORT - PACIFIC OCEAN - 1960 TYPHOON REPORT - PACIFIC OCEAN - 1961

TYPHOON REPORT - PACIFIC OCEAN - 1962

TYPHOON REPORT - PACIFIC OCEAN - 1963

$01802-003+$ $1803-003+$
$01804-003+$

$01805-004+$

TYPHOON REPORT - PACIFIC OCEAN - 1965

$01806-003+$

$01807-003+$

( $F I R E)$

-.... TEMPERATURE.

FIRE S, LIQUID HYDROCARBON FUEL, HEAT TRANSFER IN

BURNING CONDITIONS OF PETROLEUM LIQUIDS
UNC $\quad 00517-011+$ UNC $00518-001$

( $F$ IRE) ...... TEMPERATURE... . INPUT.....

AIRCRAFT ACCIDENT ENVIRONMENT - TAC STUDY
CIVIL AVIATION ACCIDENT DATA - 1962 TO 1972

CIVIL AVIATION ACCIDENT DATA- 1962
FIRE INFORMATION AIRCRAFT ACCIDENTS

A/C ACCIDENT MARTIN 404 OCT 1970

AIC ACCIDENT CONVAIR 580 DEC 1968

A/C ACCIDENT DOUGLAS C540 APRIL 1970

AIC ACCIDENT 74712 SEPT 1970

A/C ACCIDENT DC 8 63F SEPT 1970

AI RCRAFT ACCIDENT REPORTS - NTSB - 1972

AIRCRAFT ACCIDENTS, TYPE, RATE, NTSB 1970

AI RCRAFT ACCIDENTS, TYPE, RATE, NTSB 1970

AIRCRAFT ACCIDENTS, TYPE, RATE, NTSB 1973

AIRCRAFI ACCIDENT - BOEING $707-1116174$

AIRCRAFT ACCIDENT - OC $8-6 / 20173$

A/C CRASH,BT J7, BOSTON, 3 NOV 1973

$\begin{array}{ll}\text { UNC } & 11630-004+ \\ \text { UNC } & 01664-009+ \\ \text { UNC } & 01674-002+ \\ \text { UNC } & 01677-003+ \\ \text { UNC } & 01678-003+ \\ \text { UNC } & 01680-003+ \\ \text { UNC } & 01685-003+ \\ \text { UNC } & 01686-003+ \\ \text { UNC } & 01724-002+ \\ \text { UNC } & 01756-002+ \\ \text { UNC } & 01757-001+ \\ \text { UNC } & 01800-001+ \\ \text { UNC } & 01821-001+ \\ \text { UNC } & 01843-023+\end{array}$

MOTOR CARRIER ACCIDENT ENVIRONMENT - TAC STUDY

FI RE TEMPERATURES - TRUCK

ACCI DENT REPORT, IRUCK - VENTURA, CALIFORNIA, 8-18-71

TRUCK ACCIDENT-VIRGINIA 4/13/72

TRUCK ACCIDENT-PENN-9/5/71

TRUCK ACCIDENT-TENN-5/13/72

TRUCK ACCIDENT REPORTS, NHTSA, NO. 8

HYDROGEN TRANSPORT AND HANDLING EXPLOSION

TRUCK ACCIDENT - VIRGINIA - 4/13/72

TRUCK ACCIOENT - TEXAS - 3/7173

TRUCK ACCIDENT - MASSACHUSETTS - $10 / 18 / 73$

AC CI DENTS, CANADA, RAM CONTAINERS

CAR ACCIDENT, NHTSA, VOL.6, NO.11

$\begin{array}{ll}\text { UNC } & A 1662-007+ \\ \text { UNC } & 00520-001+ \\ \text { UNC } & 01659-003+ \\ \text { UNC } & 01692-003+ \\ \text { UNC } & 01693-003 \text { * } \\ \text { UNC } & 01697-002+ \\ \text { UNC } & 01754-004 \text { * } \\ \text { UNC } & 01758-001+ \\ \text { UNC } & 01784-002+ \\ \text { UNC } & 01785-002+ \\ \text { UNC } & 01788-002+ \\ \text { UNC } & 01888-013+ \\ \text { UNC } & 01898-005 \text { * }\end{array}$




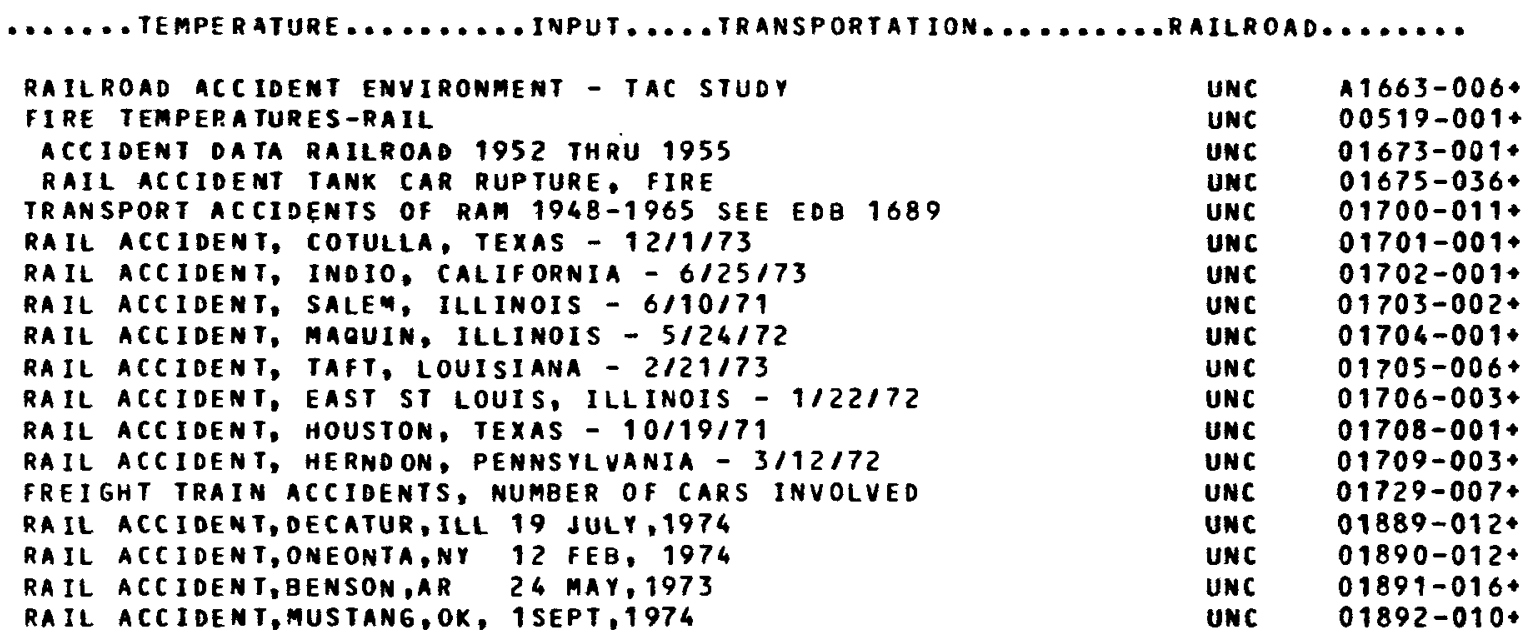

(FIRE)

(F I RE)

(FIRE)

(FI RE)
SHIP..........

UNC $\quad 00521-001+$

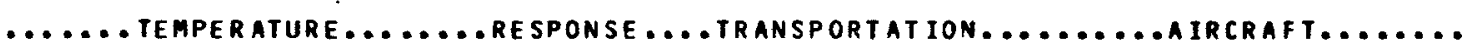

A/C CRASH,B707, BOSTON, 3 NOV 1973

UNC $01843-023+$

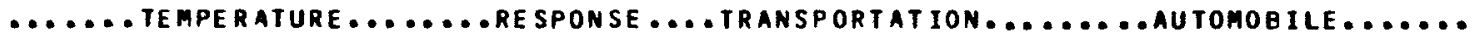

CARGO RESPONSE TRUCK AND RAIL ACCIDENTS $\quad$ UNC $01688-002+$ AC CI DENTS, CAMADA, RAM CONTAINERS

$01688-002+$

CAR ACCIDENT, NHTSA,VOL.6, NO.11

$\begin{array}{ll}\text { UNC } & 01689-011+ \\ \text { UNC } & 01888-013+\end{array}$

UNC $01898-005$.

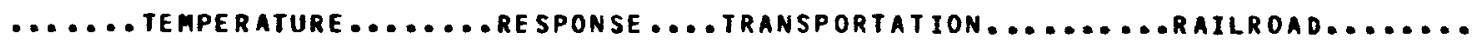

CARGO RESPONSE TRUCK ANO RAIL ACCCIDENTS SEE EDB1688

RAIL ACCIDENT, DECATUR, ILL 19 JULY, 1974

RAIL ACCIDENT, ONEONTA, NY 12 FEB, 1974

RAIL ACCIDENT,BENSON,AR 24 MAY, 1973

RAIL ACCIDENT, MUSTANG, OK, ISEPT, 1974

UNC 01699-002*

UNC 01889-012+

UNC $01890-012+$

UNC $01891-016$.

UNC $01892-010+$ 
......... In

TROPICAL CYCLONES

UNC $\quad 01856-002+$

.........HIND............ INPUT ..... TRANSPORTATION........AIRCRAFT......

HELI COPTER DOWNWASH DATA

UNC

$01760-001+$

\begin{tabular}{|c|c|c|c|c|c|c|}
\hline TORNADOS & $\therefore$ FREQL & UENCY AND & LOCATION & - UNITEO STATES & UNC & $01541-011+$ \\
\hline WI ND AND & WAVE H & HEIGHIS - & TROPICAL & STORMS & UNC & $01636-002+$ \\
\hline TYPHOON & REPORT & - PACIFIC & OCEAN - & 1959 & UNC & $01802-003+$ \\
\hline TYPHOON & REP ORT & - PACIFIC & OCEAN - & 1900 & UNC & $01803-003+$ \\
\hline TYPHOON & REPORT & - PACIFIC & OCEAN - & 1961 & UNC & $01804-003+$ \\
\hline TYPHOON & REPORT & - PACIFIC & OCEAN - & 1962 & UNC & $01805-004+$ \\
\hline TYPHOON & REPORT & - PACIFIC & OCEAN - & 1963 & UNC & $01806-003+$ \\
\hline TYPHOON & REPORT & - PACIFIC & OCEAN - & 1965 & UNC & $01807-0034$ \\
\hline TYPHOON & REPORT & - PACIFIC & OCEAN - & 1966 & UNC & $01808-003+$ \\
\hline TYPHOON & REPORT & - PACIFIC & OCEAN - & 1967 & UNC & $01809-003+$ \\
\hline TYPHOON & REPORT & - PACIFIC & OCEAN - & 1968 & UNC & $01810-004+$ \\
\hline TYPHOON & REPORT & - PACIFIC & OCEAN - & 1969 & UNC & $01819-002+$ \\
\hline TYPHOON & REPORT & - PACIFIC & OCEAN - & 1970 & UNC & $01812-003+$ \\
\hline TYPHOON & REPORT & - PACIFIC & OCEAN - & 1974 & UNC & $01813-003+$ \\
\hline
\end{tabular}


DIST RIBUTION:

TID-4500, UC-71 (134)

Transportation Branch (50)

ERDA

Division of Environmental Control Technology

Washington, DC 20545

National Science Foundation

2101 Constitution Avenue, NW

Washington, DC 20418

Attn: W. M. Carey

A. Grella (3)

DOT

Office of Hazardous Materials

Room 6218, Buzzard's Point Building 2100 2nd SW

Washington, DC 20590

L. Benner

Chief

Hazardous Materıals Safety Dıvısion NTSB

Washington, DC 20594

J. Russell

Environmental Protection Agency

Office of Radiation Programs

401 M Street, SW

Washington, DC 20460

W. F. Black

DOT

Federal Ral Administration

Trans Point Building

Washington, DC 20590

J. Buchanan

Nuclear Safety Information Center

P. O. Box Y

Oak Ridge, TN 37830

E. E. Kıdwell

DOT

Federal Highway Administration

DOT Bullding

Room 3408

Washington, DC 20590

W. E. Scott

National Highway Traffic Safety Administration

Office of Accident Investigation and Data

Analysis

Nassif Building

4007 th Street, SW

Washington, DC 20590

Mr. M. B. Gens

8027 Aspen NE

Albuquerque, NM 87110

Mr. S. G. Guins

4496 Doble Road

Okemos, MI 48864
G. A. Fowler

W. A. Gardner

T. B. Lane

W. F. Hartman

T. G. Priddy

H. C. Hardee

D. W. Larson

R. T. Othmer

E. L. Harley

J. T. Foley (10)

C. A. Davidson

D. B. Shuster

S. W. Key

O. E. Jones

V. E. Blake, Jr.

M. R. Madsen

J. T. Risse

E. I. Bruce

R. L. Peurifoy, Jr.

A. W. Snyder

R. M. Jefferson

R. E. Nickell

J. K. Cole

R. E. Luna

E. A. Aas (2)

C. A. Pepmueller (Actg) (5)

W. L. Garner (3)

For ERDA/TIC (Unlımited Release) 